(Wordcount: 8,759)

\title{
Fixed-Flowrate Total Water Network Synthesis under Uncertainty with Risk Management
} Cheng Seong Khor ${ }^{1,2}$, Benoit Chachuat ${ }^{1}$, Nilay Shah ${ }^{1}$

${ }^{1}$ Centre for Process Systems Engineering (CPSE), Imperial College London, South Kensington Campus, London SW7 2AZ, United Kingdom.

${ }^{2}$ Chemical Engineering Department, Universiti Teknologi PETRONAS, Bandar Seri Iskandar, 31750 Tronoh, Perak, Malaysia.

\begin{abstract}
This work addresses the problem of integrated water network synthesis under uncertainty with risk management. We consider a superstructure consisting of water sources, regenerators, and sinks that leads to a mixed-integer quadratically-constrained quadratic program (MIQCQP) for a fixed-flowrate total water network synthesis problem. Uncertainty in the problem is accounted for via a recourse-based two-stage stochastic programming formulation with discrete scenarios that gives rise to a multiscenario MIQCQP comprising network design in the first stage and its operation in the second stage acting as recourse. In addition, we extend the model to address risk management using the Conditional Value-at-Risk (CVaR) metric. Because a large number of scenarios are often required to capture the underlying uncertainty of the problem, causing the model to suffer from the curse of dimensionality, we propose a stepwise solution strategy to reduce the computational load. We illustrate this methodology on a case study inspired from the water network of a petroleum refinery in Malaysia. The presence of nonconvex bilinear terms necessitates the use of global optimization techniques for which we employ a new global
\end{abstract}


MIQCQP solver, GAMS/GloMIQO and verify the solutions with BARON. Our computational results show that total water network synthesis under uncertainty with risk management problems can be solved to global optimality in reasonable time.

\begin{abstract}
Keywords: water network; uncertainty; fixed-flowrate; multiscenario; mixed-integer nonlinear program (MINLP); Conditional Value-at-Risk (CVaR)
\end{abstract}

\title{
1 Introduction
}

The optimal synthesis of water network presents a significant challenge for the design of process systems particularly in the face of scarcity of freshwater resources and increasingly stringent environmental regulations on effluent discharge. A specific class of the problem termed as total water network synthesis involves a simultaneous consideration of both water-using units and water (or wastewater) treatment operations (Foo, 2009). Water-using units represent water sources or sinks, including freshwater sources, with their corresponding contaminant concentrations. Water treatment or regeneration operations act as intermediate processes to reduce contaminant levels as necessary before the sources can be subject to reuse/recycle in the sinks. The goal is to synthesize a network that integrates these water-using and waterregeneration operations by optimizing a certain objective, which is typically "environomic" in nature, i.e., as based on economics in maximizing profit or minimizing cost as well as meeting certain environmental sustainability criteria, while complying with constraints on the water users and/or final discharge limits to the environment. 
In our earlier work (Khor et al., 2012b), a deterministic fixed-flowrate formulation of the water network synthesis problem is presented that assumes fixed values of all model parameters. However, in actual operating conditions, there are often significant variations or stochastics in the parameter values. Indeed, literature data on effluent quality in process plants typically indicates significant variability in the regenerator efficiency for contaminant removals (Tchobanoglous et al., 2004). Figure 1 displays a representative trend of such removal efficiencies for six contaminants over a duration of one month as sampled to compare the influent and effluent of a reverse osmosis skid at a petroleum refinery in Malaysia (Khor et al., 2009). As evidenced from the plot, there are substantial variations in the removal ratio parameter of such a membrane regenerator unit, thus it is imperative to account for uncertainty in this parameter in a model formulation. In general, physical reasons contributing to uncertain contaminant removals are mainly due to fluctuations in operating condition as a result of fouling and leaks (resulting from ageing) in pipelines. For a membrane regenerator unit, such physical phenomena may lead to frictional pressure loss in the membrane channel and pressure drop in the module manifolds with consequential varying operating conditions (Maskan et al., 2000). Note that these sources of uncertainty are externally imposed, i.e., they are exogenous (as opposed to endogenous) uncertainty. 
Figure 1. Typical trends in fluctuations of contaminant removal efficiencies as measured at a plant over a duration of one month (source: Khor et al. (2009))

Nonetheless, only a small fraction of work in the literature to date considers the arguably more practical problem of water network synthesis under uncertainty. The work by Koppol and Bagajewicz (2003) represents a novel attempt at addressing uncertainty in water network synthesis. A mixed-integer linear program (MILP) is proposed that adopts a discrete scenario generation approach using bounded uniform distribution to represent uncertainty in contaminant mass load of water-using units and coupled with a scenario reduction technique. Additionally, the model considers financial risk management on the total network cost. The authors advocate that it is not possible to mitigate risk when the operating cost is much larger than the capital cost because a design with minimum expected operating cost usually poses minimum risk, but when capital cost is comparable to operating cost, reuse of wastewater is amenable to reducing risk. 


\begin{abstract}
Al-Redhwan et al. (2005) employs two-stage stochastic programming to formulate a fixed-load water network model with uncertain mass load parameter. Karuppiah and Grossmann (2008) also utilizes a similar framework with consideration for an additional uncertain parameter in the contaminant removal ratio. A major contribution of this work is to globally optimize the nonconvex mixed-integer nonlinear program (MINLP) by using relaxation via McCormick's (1976) convex and concave envelopes for the bilinear terms and linear overestimators constructed from secants for concave terms in the objective function. The authors propose a spatial branch-and-cut scheme with a Lagrangean decomposition approach to solve the multiscenario model.
\end{abstract}

Tan et al. (2007) utilizes a Monte Carlo simulation approach to investigate the sensitivity of solutions obtained from water pinch analysis by accounting for uncertainty in the mass load parameter. It is found that fluctuations in processing conditions can lead to process disruptions and affect product quality and process stability. In another work considering mass load uncertainty, Zhang et al. (2009) attempts to develop a resilient water network by aiming for a low value of a metric introduced as the tolerance amount of a water-using unit, which measures the difference between the limiting and actual mass load of a network. Feng et al. (2011) addresses the design of a multicontaminant water-using network with consideration for mass load uncertainty. The proposed NLP-based approach adjusts stream flows through a combination of optimization and heuristics while preserving the optimal water network structure obtained under nominal condition for minimum freshwater use. 
Hung and Kim (2011) consider uncertain inlet flowrate and mass load of water-using units. The uncertain parameters are represented as average values via a multiperiod MINLP formulation. The model addresses uncertainty by incorporating buffer tanks to handle sudden contaminant level rise and supplementary pipelines to supply freshwater to ensure feasibility. An insightsbased decomposition strategy that involves iteratively solving a sequence of MILP and LP relaxations by fixing the concentration and flowrate of water-using units, respectively is proposed to initialize the solution.

Synthesis of water regeneration systems particularly within the context of a total water network system investigates options for regeneration-reuse besides direct reuse or recycle. Tan et al. (2009) considers the use of partitioning regenerators specifically membrane technologies to 
Table 1. Some representative work on optimal water network synthesis under uncertainty

\begin{tabular}{llll}
\hline \multicolumn{1}{c}{ Reference } & \multicolumn{1}{c}{ Model Formulation } & $\begin{array}{c}\text { Uncertain Parameter and } \\
\text { Representation }\end{array}$ & \multicolumn{1}{c}{ Solution Strategy } \\
\hline $\begin{array}{l}\text { Koppol and Bagajewicz } \\
\text { (2003) }\end{array}$ & $\begin{array}{l}\text { MILP with financial risk } \\
\text { management }\end{array}$ & $\begin{array}{l}\text { Contaminant mass load } \\
\text { using discrete scenarios }\end{array}$ & $\begin{array}{l}\text { Branch-and-bound with } \\
\text { CPLEX }\end{array}$ \\
Al-Redhwan et al. (2005) & Two-stage stochastic NLP & $\begin{array}{l}\text { Contaminant mass load } \\
\text { using discrete scenarios }\end{array}$ & $\begin{array}{l}\text { Local optimization using } \\
\text { generalized reduced } \\
\text { gradient (with CONOPT2) }\end{array}$ \\
& Water pinch analysis & $\begin{array}{l}\text { Mass load using } \\
\text { discretely-generated } \\
\text { values et al. (2007) }\end{array}$ & $\begin{array}{l}\text { Iterative-based heuristics } \\
\text { using Monte Carlo } \\
\text { simulation }\end{array}$ \\
$\begin{array}{l}\text { Karuppiah and Grossmann } \\
\text { (2008) }\end{array}$ & Two-stage stochastic & $\begin{array}{l}\text { Contaminant mass load } \\
\text { and removal ratio using } \\
\text { discrete scenarios }\end{array}$ & $\begin{array}{l}\text { Global optimization using } \\
\text { branch-and-cut (with } \\
\text { BARON and DICOPT) }\end{array}$
\end{tabular}
reverse osmosis network in a fixed-flowrate total water network synthesis MINLP formulation. Sotelo-Pichardo et al. (2011) address the retrofit of a total water network by applying reconfiguration strategies that include repiping, modifying the capacity and performance of existing water-using units, and reuse of existing regenerators as well as installing new regenerators. A recent work by Tokos et al. (2013) employs a biobjective MINLP optimization model that accounts for both economic and environmental impacts of batch and semi-continuous total water network systems. A Pareto curve of optimal solution is obtained by implementing variants of the weighted-sum method. On the other hand, Lim et al. (2013) propose a reformulation approach for biobjective to single objective total water network synthesis problems that are incorporated with eco-design principles.

Table 1 summarizes the salient features of some representative work on optimal water network synthesis under uncertainty.

optimize water allocation involving multiple water sources and sinks in an MILP model. Khor et al. (2011) incorporates a detailed nonlinear membrane regenerator model as exemplified for a synthesis under uncertainty. 
It is the aim of this paper to investigate the problem of fixed-flowrate water network synthesis under uncertain conditions by extending the formulation presented in Khor et al. (2012b). The formulation employs the classical recourse-based two-stage stochastic program and uses discrete scenarios to approximate the underlying probability distribution of the uncertain removal ratio parameters. The two-stage framework comprises network design in the first stage and its operation in the second stage acting as recourse. The resulting formulation gives rise to a multiscenario MINLP, or more specifically, a mixed-integer quadratically-constrained quadratic program (MIQCQP).

The paper also addresses risk management in view of the possibility of high costs of freshwater and wastewater treatment in view of the deregulation and privatization taking place in water resources management (Bakker, 2010). The situation is compounded with increasingly stressed 
water resources globally due to growing population, pollution, inefficient irrigation, etc. (International Water Management Institute (IWMI), 2007). Consequently, a water network is exposed to higher costs if more freshwater is demanded and/or more wastewater needs treatment to meet operating and regulation requirements. According to a 2008 report by the United Nations Environment Programme (UNEP, 2008), industry is the second largest user of freshwater worldwide after agriculture (Zimmerman et al., 2008). Hence, there are practical reasons to enhance our water network synthesis formulation to consider risk management. We strive to meet this purpose by appending the Conditional Value-at-Risk $(\mathrm{CVaR})$ metric to the objective function. However, a large number of scenarios are often required to capture the underlying uncertainty of the problem, causing the model to suffer from the curse of dimensionality. To circumvent this issue, we adopt a stepwise strategy that tractably solves a risk-neutral version of the formulation using a small number of scenarios to reduce the computational load in a subsequent scenario-ordering scheme for simulating the Value-at-Risk (VaR) parameter. The strategy also serves to provide initial values for solving the nonlinear risk-averse mean-CVaR model.

The presence of nonconvex bilinear terms necessitates the implementation of a global optimization approach to ensure reliable solutions. In this regard, we solve both the risk-neutral and risk-averse formulations using a new global MIQCQP solver, GAMS 24.0.2/GloMIQO 2.1 (Misener and Floudas, 2012). We also verify the solutions with GAMS 24.0./BARON 11.9.1 (Tawarmalani and Sahinidis, 2005), a state-of-the-art commercial global solver. 
The rest of the article is organized as follows. Section 2 formally describes the problem under uncertainty which we wish to address, while Section 3 proceeds to outline in general, our proposed optimization-based framework with a computationally-tractable solution strategy. Then, we present formulations for the risk-neutral and risk-averse problems of water network synthesis as two-stage stochastic programs in Section 4. Section 5 reports computational results using the two commercial solvers before concluding the paper in Section 6.

\section{Problem Statement}

This work aims to optimally synthesize a total water network, which integrates both water-using and water treatment operations, as given the following elements of a water network:

- a set of fixed-flowrate water sources $i, i \in I$, with known flowrates $F_{\text {SO }}(i)$ and concentrations $C_{\mathrm{SO}}(i, q)$ of the contaminants $q \in Q$;

- a set of fixed-flowrate water sinks $j, j \in J$, with known flowrate requirements $F_{\mathrm{SI}}(j)$ and maximum allowable inlet concentration limit $C^{\max }(j, q)$ of the contaminants $q$;

- a set of water regenerators $k, k \in K$, with uncertain removal ratios of contaminants; and

- a freshwater source $i=\mathrm{FW}$ with known contaminant concentrations that can be purchased to supplement available water sources.

\subsection{Uncertainty Description}


As elucidated, uncertainty in water network synthesis primarily concerns contaminant removal ratios in the regeneration subnetwork. Removal ratio or removal efficiency $R(k, q, s)$ of a targeted contaminant $q$ for a regenerator $k$ is defined as the amount $q$ (expressed in ratio or percentage) removed by $k$ from an incoming water stream under an operating scenario $s$. The uncertain removal ratio parameters can be assumed to be represented by a bounded uniform probability distribution within a certain known interval defined by its lower and upper bounds. This assumption is reasonable because it is possible to specify the range within which removal ratios vary. In our model, we treat the removal ratios as random variables that may take on multiple possible discrete values under different network operation scenarios. For this purpose, we sample random values of the removal ratios from a known discrete probability distribution using a Monte Carlo-simulation based technique.

\subsection{Two-Stage Stochastic Programming Framework}

Fluctuations in the removal amounts, as exemplified by Figure 1, may consequently necessitate a recourse action of adjusting freshwater supply for diluting flows to the regenerators and the sinks. Such a recourse action ensures that the network may still be operated without violating the maximum allowable inlet concentration (MAIC) limits of the sinks, particularly for discharge limits that are bounded by strict environmental regulations. In this regard, another major recourse action involves regulating wastewater flows to the effluent treatment system (ETS) to comply with discharge limits. 
The decision variables are divided into first and second stages corresponding to before and after information on the uncertainty have been revealed, respectively. Similar to the approach of Karuppiah and Grossmann (2008), the first-stage decisions pertain to determining the optimal network topology in terms of interconnections of the entities comprising sources, regenerators, and sinks. These decisions are represented by two types of design variables, which have to be taken prior to revelation of uncertainty in the system. They are the $0-1$ variables $y_{\mathrm{A}, \mathrm{B}}(\alpha, \beta)$ describing existence of the interconnections between an origin entity A (as indexed by $\alpha$ ) and a destination entity B (as indexed by $\beta$ ), as well as the continuous variables on the maximum allowable total stream flows $F_{\mathrm{A}, \mathrm{B}}^{\max }(\alpha, \beta)$.

The second-stage decisions are recourse or corrective actions with respect to the first-stage decisions after the uncertainty is revealed. Here, the optimal operating policy of the network is established by the continuous recourse variables of flows and concentrations in each network interconnection, particularly freshwater supply and wastewater inlet flows through the regenerators, as stated earlier. These second-stage operational flows can be different during network operation in accordance with the discrete-valued scenarios assumed by the uncertain removal ratios. Thus, flow in an interconnection is sent at a rate $F_{\mathrm{A}, \mathrm{B}}(\alpha, \beta, s)$ from an origin entity A to a destination entity B for a particular realization of the operating scenario $s$, while the associated contaminant concentrations leaving and entering a regenerator $k$ for a set of contaminants $q \in Q$ is given by $C_{\mathrm{A}}(k, q, s)$. Figure 2 provides a diagrammatic representation of the proposed framework for a piping interconnection between two network elements A and B. 

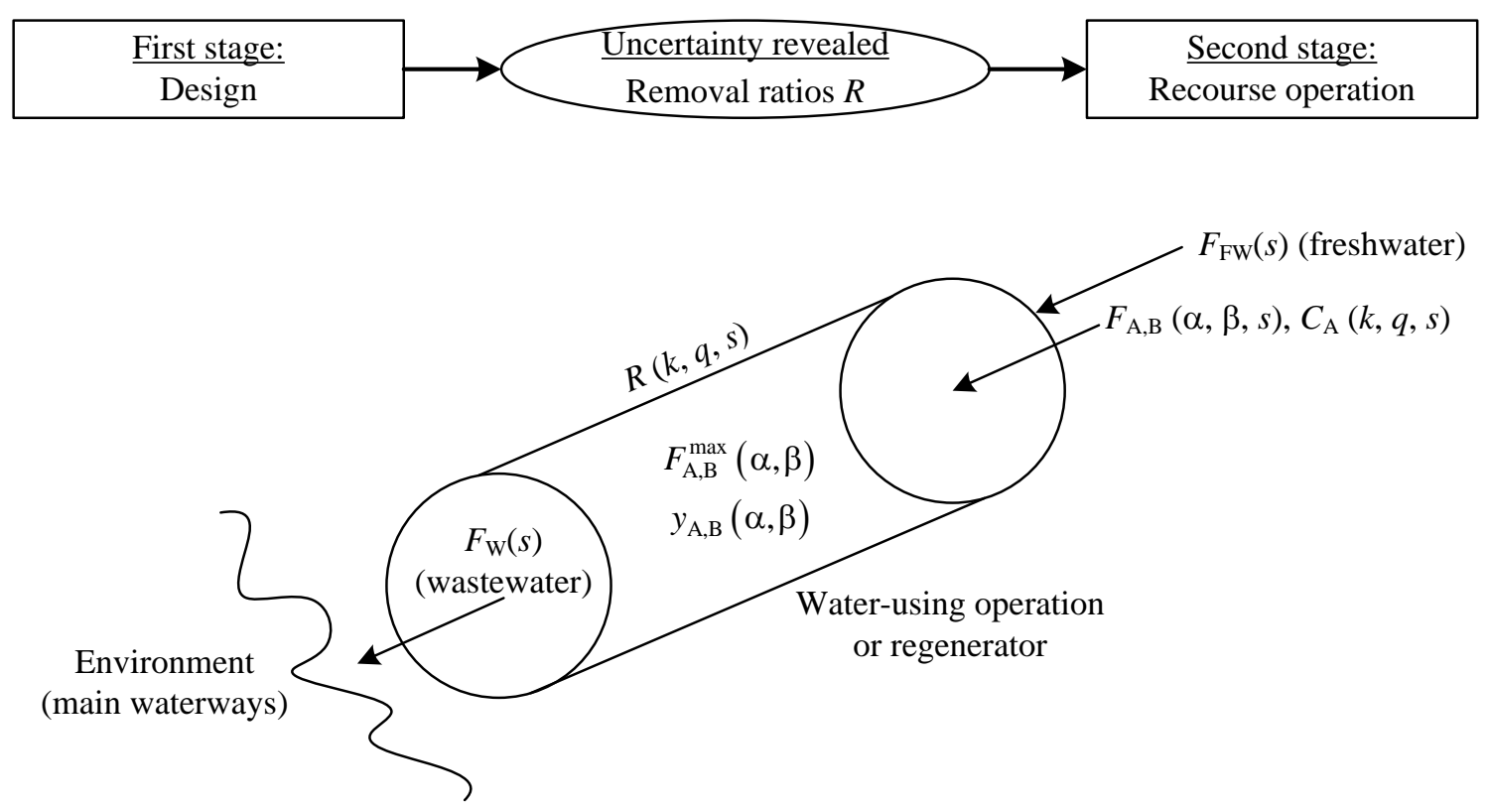

Figure 2. Illustration of proposed two-stage stochastic programming framework

Conventional two-stage stochastic programming, which exploits the underlying structure of the problem presented here, provides a suitable framework because uncertainty in removal ratios is exogenous in nature as aforementioned. Moreover, a stochastic program with complete recourse formulation (Sen and Higle, 1999) can be considered as opposed to a decision-dependent approach (Tarhan et al., 2009).

The objective of the proposed two-stage stochastic program is to synthesize an integrated water system that minimizes the total annualized cost of designing the network in the first stage and the expected cost of operating the network in the second stage. A similar framework has been adopted by Karuppiah and Grossmann (2008) for a water network synthesis problem but nonetheless for a fixed-load water-using unit representation. This modeling framework has also found recent applications in $\mathrm{Li}$ et al. (2011) for a pooling problem of natural gas production and Yunt et al. (2008) for a portable power generation system with varying demand levels. We 
emphasize again that it is at the second stage that the flexibility of the design is checked by considering variations through the recourse operating variables in accommodating the uncertain parameter realizations. This part of the model is also the most computationally demanding. It is noteworthy that the integer decisions do not appear in the second stage and hence, our formulation do not fall under the class of problems of stochastic mixed-integer recourse programs, which is treated, among others, in the work of Schultz (2003).

\section{Water Network Model Formulation}

\subsection{Superstructure Representation}

Based on the work of Khor et al. (2012a, b), we adopt a source-regenerator-sink superstructure that allows all feasible interconnections of the network entities to cater for alternative configurations involving direct water reuse/recycle, regeneration-reuse, and regenerationrecycle. Figure 3 shows a simplified superstructure around both the membrane and nonmembrane regenerators. The representation leads to a MINLP formulation by adopting the notations explained earlier as based on Figure 2. 


\subsection{Regenerator Models}

Conventional models for water regenerators typically involve a single pooled inlet stream from a source and a single pooled outlet stream to a sink (see, e.g., Karuppiah and Grossmann (2008)), which we directly employ for the non-membrane regenerators (NM). On the other hand, we adapt such a representation for the membrane regenerators $(\mathrm{M})$ that typically consist of two outlets, namely a permeator stream (MP) which is of lower concentration than a rejector stream (MR). The outlet flow of a regenerator in a conventional model would have been separated into a permeator and a rejector by using a splitter, but both resultant streams would then share the same concentration. To avoid such a possible concentration discrepancy, we represent the permeator and the rejector as units instead of streams in our proposed linear membrane regenerator model. Our proposed model also accounts for full interconnectivity among the regenerators by allowing an interconnection between a non-membrane regenerator $k$ with another non-membrane regenerator $k^{\prime}$ or a membrane regenerator $k^{\prime \prime}$ — similar cases apply for a permeator and a rejector. 


\subsection{Optimization Model Formulation}

\subsubsection{Material Balances}

\subsubsection{Water Sources}

Mass balance around a source except freshwater for each scenario is represented as:

$$
\begin{aligned}
& \sum_{k \in K_{\mathrm{NM}}} F_{\mathrm{SO}, \mathrm{NM}}(i, k, s)+\sum_{k^{\prime} \in K_{\mathrm{M}}}\left(F_{\mathrm{SO}, \mathrm{MP}}\left(i, k^{\prime}, s\right)+F_{\mathrm{SO}, \mathrm{MR}}\left(i, k^{\prime}, s\right)\right)+\sum_{j \in J} F_{\mathrm{SO}, \mathrm{SI}}(i, j, s) \\
= & F_{\mathrm{SO}}(i), \quad \forall i \in I \backslash\{\text { Freshwater }\}, \forall s \in S .
\end{aligned}
$$

For a freshwater source, its supply flowrate is a recourse variable that varies with the scenarios:

$$
\begin{aligned}
& \sum_{k \in K_{\mathrm{NM}}} F_{\mathrm{SO}, \mathrm{NM}}(i, k, s)+\sum_{k^{\prime} \in K_{\mathrm{M}}}\left(F_{\mathrm{SO}, \mathrm{MP}}\left(i, k^{\prime}, s\right)+F_{\mathrm{SO}, \mathrm{MR}}\left(i, k^{\prime}, s\right)\right)+\sum_{j \in J} F_{\mathrm{SO}, \mathrm{SI}}(i, j, s) \\
= & F_{\mathrm{FW}}(s), \quad i=\text { Freshwater, } \forall s \in S .
\end{aligned}
$$

\subsubsection{Non-Membrane Regenerators}

Mass and concentration balances around the inlet of a non-membrane regenerator: 


$$
\begin{aligned}
& \left(\begin{array}{l}
\sum_{i \in I} F_{\mathrm{SO}, \mathrm{NM}}(i, k, s) C_{\mathrm{SO}}(i, q)+\sum_{\substack{k^{\prime} \in K_{\mathrm{NM}} \\
k^{\prime} \neq k}} F_{\mathrm{NM}, \mathrm{NM}}\left(k^{\prime}, k, s\right) C_{\mathrm{NM}}\left(k^{\prime}, q, s\right) \\
+\sum_{k^{\prime \prime} \in K_{\mathrm{M}}}\left(F_{\mathrm{MP}, \mathrm{NM}}\left(k^{\prime \prime}, k, s\right) C_{\mathrm{MP}}\left(k^{\prime \prime}, q, s\right)+F_{\mathrm{MR}, \mathrm{NM}}\left(k^{\prime \prime}, k, s\right) C_{\mathrm{MR}}\left(k^{\prime \prime}, q, s\right)\right)
\end{array}\right) \\
& =F_{\mathrm{NM}}^{\mathrm{F}}(k, s) C_{\mathrm{NM}}^{\mathrm{F}}(k, q, s), \quad \forall k \in K_{\mathrm{NM}}, \forall q \in Q, \forall s \in S .
\end{aligned}
$$

where $F_{\mathrm{NM}}^{\mathrm{F}}(k, s)$ is the mass flowrate of the feed stream to a non-membrane regenerator.

$$
\begin{aligned}
& \text { Mass and concentration balances around the outlet of a non-membrane regenerator: } \\
& \sum_{j \in J} F_{\mathrm{NM}, \mathrm{SI}}(k, j, s)+\sum_{\substack{k^{\prime} \in K_{\mathrm{NM}} \\
k^{\prime} \neq k}} F_{\mathrm{NM}, \mathrm{NM}}\left(k, k^{\prime}, s\right)+\sum_{k^{\prime \prime} \in K_{\mathrm{M}}}\left(F_{\mathrm{NM}, \mathrm{MP}}\left(k, k^{\prime \prime}, s\right)+F_{\mathrm{NM}, \mathrm{MR}}\left(k, k^{\prime \prime}, s\right)\right) \\
& =F_{\mathrm{NM}}^{\mathrm{F}}(k, s), \quad \forall k \in K_{\mathrm{NM}}, \forall s \in S . \\
& \quad(1-R(k, q, s)) C_{\mathrm{NM}}^{\mathrm{F}}(k, q)=C_{\mathrm{NM}}(k, q), \quad \forall k \in K_{\mathrm{NM}}, \forall q \in Q, \forall s \in S .
\end{aligned}
$$

Recall that $R(k, q, s)$ is a random variable representing the uncertain parameter of removal ratio. It is noteworthy that the uncertainty appears as a coefficient on the left-hand side of the constraints. 


\subsubsection{Membrane Regenerators}

Mass and concentration balances around entry point to the permeator of a membrane regenerator:

$$
\begin{aligned}
& \sum_{i \in I} F_{\mathrm{SO}, \mathrm{MP}}(i, k, s)+\sum_{k^{\prime} \in K_{\mathrm{NM}}} F_{\mathrm{NM}, \mathrm{MP}}\left(k^{\prime}, k, s\right)+\sum_{\substack{k^{\prime \prime} \in K_{\mathrm{M}} \\
k^{\prime \prime} \neq k}}\left(F_{\mathrm{MP}, \mathrm{MP}}\left(k^{\prime \prime}, k, s\right)+F_{\mathrm{MR}, \mathrm{MP}}\left(k^{\prime \prime}, k, s\right)\right) \\
& =F_{\mathrm{M}}^{\mathrm{F}}(k, s), \quad \forall k \in K_{\mathrm{M}}, \forall s \in S .
\end{aligned}
$$

Mass and concentration balances around entry point to the rejector of a membrane regenerator:

$$
\begin{aligned}
& \left(\sum_{i \in I} F_{\mathrm{SO}, \mathrm{MR}}(i, k, s)+\sum_{k^{\prime} \in K_{\mathrm{NM}}} F_{\mathrm{NM}, \mathrm{MR}}\left(k^{\prime}, k, s\right)+\sum_{\substack{k^{\prime \prime} \in K_{\mathrm{M}} \\
k^{\prime \prime} \neq k}}\left(F_{\mathrm{MP}, \mathrm{MR}}\left(k^{\prime \prime}, k, s\right)+F_{\mathrm{MR}, \mathrm{MR}}\left(k^{\prime \prime}, k, s\right)\right)\right) \\
& =F_{\mathrm{M}}^{\mathrm{F}}(k, s), \quad \forall k \in K_{\mathrm{M}}, \forall s \in S . \\
& \left(\begin{array}{l}
\sum_{i \in I}\left(F_{\mathrm{SO}, \mathrm{MR}}(i, k, s)\right) C_{\mathrm{SO}}(i, q)+\sum_{k^{\prime} \in K_{\mathrm{NM}}} F_{\mathrm{NM}, \mathrm{MR}}\left(k^{\prime}, k, s\right) C_{\mathrm{NM}}\left(k^{\prime}, q, s\right) \\
+\sum_{\substack{k^{\prime \prime} \in K_{\mathrm{M}} \\
k^{\prime \prime} \neq k}} F_{\mathrm{MP}, \mathrm{MR}}\left(k^{\prime \prime}, k, s\right) C_{\mathrm{MP}}\left(k^{\prime \prime}, q, s\right)+\sum_{\substack{k^{\prime \prime} \in K_{\mathrm{M}} \\
k^{\prime \prime} \neq k}} F_{\mathrm{MR}, \mathrm{MR}}\left(k^{\prime \prime}, k, s\right) C_{\mathrm{MR}}\left(k^{\prime \prime}, q, s\right)
\end{array}\right) \\
& =F_{\mathrm{M}}^{\mathrm{F}}(k, s) C_{\mathrm{M}}^{\mathrm{F}}(k, q, s), \quad \forall k \in K_{\mathrm{M}}, \forall q \in Q, \forall s \in S .
\end{aligned}
$$


It is noted that the mass flowrate of an entry point to a membrane regenerator is represented as $F_{\mathrm{M}}^{\mathrm{F}}(k, s)$ without being distinguished by a subscript denoting a permeator or a rejector. This is because physically, the permeator and the rejector are entities of the same membrane regenerator unit, hence careful formulation has been employed to maintain consistency with the actual physical configuration, which also lends a more natural formulation to the underlying problem.

Mass and concentration balances around the permeator of a membrane regenerator:

$$
\begin{aligned}
& \sum_{j \in J} F_{\mathrm{MP}, \mathrm{SI}}(k, j, s)+\sum_{k^{\prime} \in K_{\mathrm{MM}}} F_{\mathrm{MP}, \mathrm{NM}}\left(k, k^{\prime}, s\right)+\sum_{\substack{k^{\prime \prime} \in K_{\mathrm{M}} \\
k^{\prime \prime} \neq k}}\left(F_{\mathrm{MP}, \mathrm{MP}}\left(k, k^{\prime \prime}, s\right)+F_{\mathrm{MP}, \mathrm{MR}}\left(k, k^{\prime \prime}, s\right)\right) \\
& =\lambda(k) F_{\mathrm{M}}^{\mathrm{F}}(k, s), \quad \forall k \in K_{\mathrm{M}}, \forall s \in S \\
& \quad(1-R(k, q, s)) C_{\mathrm{M}}^{\mathrm{F}}(k, q, s)=\lambda(k) C_{\mathrm{MP}}(k, q, s), \quad \forall k \in K_{\mathrm{M}}, \forall q \in Q, \forall s \in S .
\end{aligned}
$$

Mass and concentration balances around the rejector of a membrane regenerator:

$$
\begin{aligned}
& \sum_{j \in J} F_{\mathrm{MR}, \mathrm{SI}}(k, j, s)+\sum_{k^{\prime} \in K_{\mathrm{NM}}} F_{\mathrm{MR}, \mathrm{NM}}\left(k, k^{\prime}, s\right)+\sum_{\substack{k^{\prime} \in K_{\mathrm{M}} \\
k^{\prime} \neq k}}\left(F_{\mathrm{MR}, \mathrm{MP}}\left(k, k^{\prime \prime}, s\right)+F_{\mathrm{MR}, \mathrm{MR}}\left(k, k^{\prime \prime}, s\right)\right) \\
& =(1-\lambda(k)) F_{\mathrm{M}}^{\mathrm{F}}(k, s), \quad \forall k \in K_{\mathrm{M}}, \forall s \in S .
\end{aligned}
$$

$$
R(k, q, s) C_{\mathrm{M}}^{\mathrm{F}}(k, q, s)=(1-\lambda(k)) C_{\mathrm{MR}}(k, q, s), \quad \forall k \in K_{\mathrm{M}}, \forall q \in Q, \forall s \in S .
$$


where $\lambda(k)$ is the split ratio on flow as based on the liquid phase recovery factor for the permeator.

\subsubsection{Sinks}

Mass balance (linear) around a sink except the discharge:

$$
\begin{aligned}
& \sum_{i \in I} F_{\mathrm{SO}, \mathrm{SI}}(i, j, s)+\sum_{k \in K_{\mathrm{NM}}} F_{\mathrm{NM}, \mathrm{SI}}(k, j, s)+\sum_{k^{\prime} \in K_{\mathrm{M}}}\left(F_{\mathrm{MP}, \mathrm{SI}}\left(k^{\prime}, j, s\right)+F_{\mathrm{MR}, \mathrm{SI}}\left(k^{\prime}, j, s\right)\right) \\
& =F_{\mathrm{SI}}(j), \quad \forall j \in J \backslash\{\text { Discharge }\}, \forall s \in S .
\end{aligned}
$$

The discharge flowrate is a recourse variable that is dependent on the scenarios:

$$
\begin{aligned}
& \sum_{i \in I} F_{\mathrm{SO}, \mathrm{SI}}(i, j, s)+\sum_{k \in K_{\mathrm{NM}}} F_{\mathrm{NM}, \mathrm{SI}}(k, j, s)+\sum_{k^{\prime} \in K_{\mathrm{M}}}\left(F_{\mathrm{MP}, \mathrm{SI}}\left(k^{\prime}, j, s\right)+F_{\mathrm{MR}, \mathrm{SI}}\left(k^{\prime}, j, s\right)\right) \\
& =F_{\mathrm{W}}(s), \quad j=\text { Discharge, } \forall s \in S .
\end{aligned}
$$

\subsubsection{Quality Constraints}

Constraint on the quality requirement for a sink as stipulated by its maximum allowable inlet concentration (MAIC) limit for a contaminant is given by: 


$$
\begin{aligned}
& \sum_{i \in I} F_{\mathrm{SO}, \mathrm{SI}}(i, j, s) C_{\mathrm{SO}}(i, q)+\sum_{k \in K_{\mathrm{NM}}} F_{\mathrm{NM}, \mathrm{SI}}(k, j, s) C_{\mathrm{NM}}(k, q, s) \\
& +\sum_{k^{\prime} \in K_{\mathrm{M}}}\left(F_{\mathrm{MP}, \mathrm{SI}}\left(k^{\prime}, j, s\right) C_{\mathrm{MP}}\left(k^{\prime}, q, s\right)+F_{\mathrm{MR}, \mathrm{SI}}\left(k^{\prime}, j, s\right) C_{\mathrm{MR}}\left(k^{\prime}, q, s\right)\right) \\
& \leq F_{\mathrm{SI}}(j) C^{\max }(j, q), \quad \forall j \in J, \forall q \in Q, \forall s \in S
\end{aligned}
$$

where $C^{\max }(j, q)$ is MAIC for a regenerator $k$ for each of the contaminants $q$. For a discharge sink, its quality constraint is given by:

$$
\begin{aligned}
& \sum_{i \in I} F_{\mathrm{SO}, \mathrm{SI}}(i, j, s) C_{\mathrm{SO}}(i, q)+\sum_{k \in K_{\mathrm{NM}}} F_{\mathrm{NM}, \mathrm{SI}}(k, j, s) C_{\mathrm{NM}}(k, q, s) \\
& +\sum_{k^{\prime} \in K_{\mathrm{M}}}\left(F_{\mathrm{MP}, \mathrm{SI}}\left(k^{\prime}, j, s\right) C_{\mathrm{MP}}\left(k^{\prime}, q, s\right)+F_{\mathrm{MR}, \mathrm{SI}}\left(k^{\prime}, j, s\right) C_{\mathrm{MR}}\left(k^{\prime}, q, s\right)\right) \\
& \leq F_{\mathrm{W}}(s) C^{\max }(j, q), \quad j=\text { Discharge, } \forall q \in Q, \forall s \in S
\end{aligned}
$$

\subsubsection{Operational Constraints}

The operational constraints link the second-stage operating flow variables in every scenario with their corresponding first-stage design flow variables to guarantee operating feasibility. From a physical viewpoint, these constraints serve to accommodate the various possible operating policies by ensuring that the interconnections, which are essentially pipelines, are operable. Thus, they are designed in such a way that the maximum design flows have to be greater than their operational counterparts in each scenario: 


$$
\begin{gathered}
F_{\mathrm{A}, \mathrm{B}}(\alpha, \beta, s) \leq F_{\mathrm{A}, \mathrm{B}}^{\max }(\alpha, \beta), \\
\forall(\alpha, \mathrm{A}) \in(I, \mathrm{SO}) \cup\left(K_{\mathrm{NM}}, \mathrm{NM}\right) \cup\left(K_{\mathrm{M}}, \mathrm{MP}\right) \cup\left(K_{\mathrm{M}}, \mathrm{MR}\right), \\
\forall(\beta, \mathrm{B}) \in(J, \mathrm{SI}) \cup\left(K_{\mathrm{NM}}, \mathrm{NM}\right) \cup\left(K_{\mathrm{M}}, \mathrm{MP}\right) \cup\left(K_{\mathrm{M}}, \mathrm{MR}\right), \\
\forall s \in S .
\end{gathered}
$$

In addition, linear logical constraints on selection of the interconnections link their existence, as given by $y_{\mathrm{A}, \mathrm{B}}(\alpha, \beta)$, with the corresponding maximum design flows $F_{\mathrm{A}, \mathrm{B}}^{\max }(\alpha, \beta)$ :

$$
\begin{aligned}
& F_{\mathrm{A}, \mathrm{B}}^{\mathrm{L}}(\alpha, \beta) y_{\mathrm{A}, \mathrm{B}}(\alpha, \beta) \leq F_{\mathrm{A}, \mathrm{B}}^{\max }(\alpha, \beta) \leq F_{\mathrm{A}, \mathrm{B}}^{\mathrm{U}}(\alpha, \beta) y_{\mathrm{A}, \mathrm{B}}(\alpha, \beta), \\
& \forall(\alpha, \mathrm{A}) \in(I, \mathrm{SO}) \cup\left(K_{\mathrm{NM}}, \mathrm{NM}\right) \cup\left(K_{\mathrm{M}}, \mathrm{MP}\right) \cup\left(K_{\mathrm{M}}, \mathrm{MR}\right), \\
& \forall(\beta, \mathrm{B}) \in(J, \mathrm{SI}) \cup\left(K_{\mathrm{NM}}, \mathrm{NM}\right) \cup\left(K_{\mathrm{M}}, \mathrm{MP}\right) \cup\left(K_{\mathrm{M}}, \mathrm{MR}\right) .
\end{aligned}
$$

where $F_{\mathrm{A}, \mathrm{B}}^{\mathrm{L}}(\alpha, \beta)$ and $F_{\mathrm{A}, \mathrm{B}}^{\mathrm{U}}(\alpha, \beta)$ are the lower and upper bounds on maximum flows, respectively. The constraints imply that if an interconnection is optimally selected, then its design flows can take values between the specified bounds; otherwise, these values are zero.

\section{Solution Methodology}

\subsection{Two-Phase Solution Approach}

A large number of scenarios are often required to capture the underlying uncertainty of a water network synthesis problem, causing the model to suffer from the curse of dimensionality. To handle this challenge of high computational load, we employ a sequential two-phase solution approach as shown in Figure 4 in which the first phase (Phase I) generates a risk-neutral solution. 
Based on Phase I solution, the VaR parameter is estimated using a scenario-ordering procedure and subsequently utilized in Phase II to compute the intended risk-averse solution.

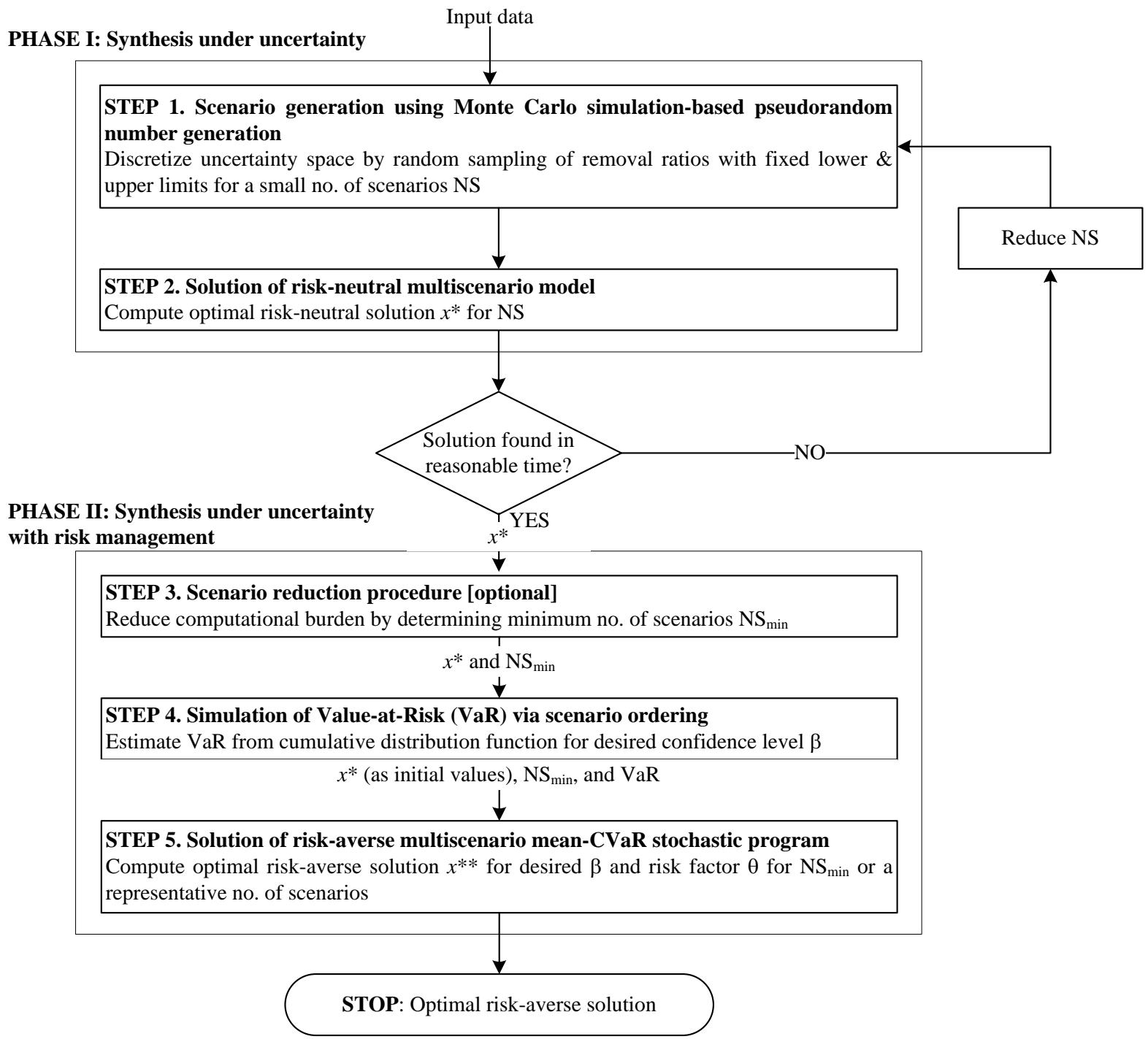

Figure 4. Proposed two-phase solution approach for water network synthesis under uncertainty with risk management 


\subsubsection{Phase I: Risk-Neutral Model}

The goal of this step is to obtain a solution with minimum cost without being averse to taking risks, which implies that the result obtained is one in which risky decisions are not penalized. Solution of such a risk-neutral multiscenario model will be used subsequently to estimate the $\mathrm{VaR}$ parameter. If a solution of the model cannot be found in reasonable time, we may consider generating a smaller but still representative set of scenarios to solve the multiscenario model.

We pose a multiscenario risk-neutral program based on our proposed model in Khor et al. (2012b) which employs a linear membrane regenerator technology representation, but reformulated to address uncertainty in the removal ratio parameters. The formulation results in a mixed-integer quadratically-constrained quadratic program (MIQCQP), a special form of mixedinteger nonlinear program (MINLP), as presented in the rest of this section. $K_{\mathrm{NM}}$ denotes the set of non-membrane regenerators, while the set $K_{\mathrm{M}}$ of membrane regenerators contains ordered pairs of the permeator MP and the rejector MR.

\subsubsection{Scenario Generation}

We first employ a Monte Carlo approach using pseudorandom number generation to generate scenarios that approximate the original full space of the probability distribution of the uncertain parameters. The values of the uncertain parameters in each scenario and their probability of occurrence are randomly sampled using pseudorandom numbers and are therefore independent of the first-stage decisions. Alternatively, the scenarios can be considered as equiprobable. In this 
regard, our approach differs from previous work (e.g., Koppol and Bagajewicz (2003)) in the sense that we consider the scenarios as constructed from random variables, instead of historical observations that may be susceptible to noisy data. As will be shown in the discussion of the numerical results in section 5 , the quality of the data that constitute the scenarios is essential to ensure a reliable solution is obtained.

A scenario is thus fully described by the values of the uncertain parameters with its associated probability, and provides a set of candidate optimal solutions exhibiting varying risk propensity. The number of all scenarios is given by the number of scenarios NS for each uncertain parameter raised to the power of the number of uncertain parameters NP, hence the problem is of combinatorial nature. In this regard, deciding a priori on the number of scenarios to be generated is a tradeoff between the computational load and the need to sufficiently capture the underlying uncertainty of the problem. This calls for a combination of the knowledge of a modeler and the experience of a decision-maker (who is typically the desired end-user) as well as past practices reported in the literature for a similar class of problems.

\subsubsection{Objective Function}

The objective function of the model involves minimizing the first-stage costs of the piping interconnections (PI) and the expected costs of the second-stage recourse operations:

$$
\min \delta \mathrm{CC}_{\mathrm{PI}}+\sum_{s \in S} p(s)\left(\mathrm{OC}_{\mathrm{PI}}(s)+\mathrm{OC}_{\mathrm{FW}}(s)+\mathrm{OC}_{\mathrm{W}}(s)\right)
$$


where $\delta$ is the annualized capital charge factor that represents tradeoffs between capital cost $\left(\mathrm{CC}_{\mathrm{PI}}\right)$ and operating cost $\left(\mathrm{OC}_{\mathrm{PI}}\right)$ of the interconnections. Components of the first-stage interconnection costs are taken as linear functions of the $0-1$ variables denoting their existences and the continuous maximum flow variables.

On the other hand, components of the expected operating costs are assumed to be linear functions of the recourse operational flows (for $\mathrm{OC}_{\mathrm{PI}}$ ), as well as cost penalties due to recourse actions involving freshwater consumption $\left(\mathrm{OC}_{\mathrm{FW}}\right)$ and wastewater treatment in the ETS $\left(\mathrm{OC}_{\mathrm{W}}\right)$. Expressions for these cost components on an annualized basis are defined as follows:

$$
\begin{gathered}
\mathrm{CC}_{\mathrm{PI}}=\sum_{(\alpha, \mathrm{A}),(\beta, \mathrm{B}) \in N} b_{\mathrm{A}, \mathrm{B}}(\alpha, \beta) F_{\mathrm{A}, \mathrm{B}}^{\max }(\alpha, \beta)+d_{\mathrm{A}, \mathrm{B}}(\alpha, \beta) y_{\mathrm{A}, \mathrm{B}}(\alpha, \beta), \\
\mathrm{OC}_{\mathrm{PI}}(s)=\sum_{(\alpha, \mathrm{A}),(\beta, \mathrm{B}) \in N} c_{\mathrm{A}, \mathrm{B}}(\alpha, \beta) F_{\mathrm{A}, \mathrm{B}}(\alpha, \beta, s),
\end{gathered}
$$

$$
\begin{gathered}
\mathrm{OC}_{\mathrm{FW}}(s)=c_{\mathrm{FW}} F_{\mathrm{FW}}(s) H, \quad \forall s \in S, \\
\mathrm{OC}_{\mathrm{W}}(s)=c_{\mathrm{W}} F_{\mathrm{W}}(s) H, \quad \forall s \in S,
\end{gathered}
$$

where $b_{\mathrm{A}, \mathrm{B}}, c_{\mathrm{A}, \mathrm{B}}, d_{\mathrm{A}, \mathrm{B}}, c_{\mathrm{FW}}$, and $c_{\mathrm{W}}$ are the respective cost coefficients. $H$ indicates hours of plant operation per annum. 


\subsubsection{Phase II: Risk-Averse Model Formulation}

The consideration for incorporating risk in a two-stage stochastic program is to account for the variability, besides the expected value, of the second-stage function as a measure of the desired intensity of the recourse operations. In this work, we adopt $\mathrm{CVaR}$ as a proxy to represent risk in the objective function. $\mathrm{CVaR}$ has received increasing applications in process systems engineering in recent work (Colvin and Maravelias, 2011; Tometzki and Engell, 2011; Verderame and Floudas, 2010; 2011).

$\mathrm{CVaR}$ is a risk measure originally employed to reduce the probability that an investment portfolio will incur high losses. It is closely related to VaR that measures the maximum expected loss in the value of a risky entity at a certain confidence interval (typically $95 \%$ or $99 \%$ ) over a given period under normal market conditions. $\mathrm{CVaR}$ is the expected loss given that the actual loss exceeds some VaR threshold at the same confidence level (Rockafellar and Uryasev, 2002; Szego, 2002). For example, at a one-month $95 \%$ confidence interval, VaR reports a single value with $95 \%$ certainty that that is the value of the maximum expected loss. CVaR measures the expectation that the value is greater than $\mathrm{VaR}$. Within a water network design setting, for instance, if $\mathrm{VaR}$ for a network cost (capital and operational) is \$1 million at a one-month $95 \%$ confidence interval, this implies that there is a $5 \%$ probability that the cost will drop more than $\$ 1$ million over any given month. CVaR is the expected loss in the network cost that is greater than \$1 million over the same duration for the same confidence interval. 
Rockafellar and Uryasev $(2000,2002)$ propose the following linear (convex) approximation of $\mathrm{CVaR}$ for a discrete probability distribution function associated with the first- and second-stage decision variables $x$ and $y(s)$, respectively, which can be interpreted as a weighted average between $\mathrm{VaR}$ and the losses exceeding VaR:

$$
\mathrm{CVaR} \approx \mathrm{VaR}+\frac{1}{1-\beta} \sum_{s=1}^{\mathrm{NS}} p(s)(f(x, y(s))-\mathrm{VaR})
$$

where $\beta$ denotes confidence level.

\subsubsection{Simulation of Value-at-Risk (VaR)}

The value of the risk measure $\mathrm{VaR}$ for a problem can be simulated in an offline manner by developing its associated cumulative distribution function (CDF) plot or risk curve (Barbaro and Bagajewicz, 2004) by using the risk-neutral solution. VaR is expressed as:

$$
\operatorname{VaR}(x, y)=\min _{x, y}\{f(x, y(s)) \mid G(f) \geq \alpha\}
$$

where $f(x, y(s))$ is the objective function of the optimization problem. $G(f)$ is the cumulative distribution function plot for the objective, and it is straightforward to determine VaR from such a plot (Santoso et al., 2005; Webby et al., 2007). 
The resultant convex mean-CVaR objective function is formulated as follows using a weighting method for examining the tradeoffs between the two conflicting objectives of expected cost and risk (Khor et al., 2011):

$$
\min \delta \mathrm{CC}_{\mathrm{PI}}+\sum_{s \in S} p(s)\left(\mathrm{OC}_{\mathrm{PI}}(s)+\mathrm{OC}_{\mathrm{FW}}(s)+\mathrm{OC}_{\mathrm{W}}(s)\right)+\theta \mathrm{CVaR}(s)
$$

where $\theta$ is a decision maker-defined adjustable non-negative weights of risk factors that facilitate the tradeoff between expected cost with risk. A larger value of $\theta$ indicates a higher propensity towards risk-taking and vice versa. In a more general setting, alternative risk measures besides CVaR such as downside risk (Eppen et al., 1989) and financial risk (Barbaro and Bagajewicz, 2004) are also applicable.

The complete formulation of a risk-averse multiscenario water network synthesis under uncertainty model is given by:

$$
\begin{array}{ll}
\min & \delta \mathrm{CC}_{\mathrm{PI}}+\sum_{s \in S} p(s)\left(\mathrm{OC}_{\mathrm{PI}}(s)+\mathrm{OC}_{\mathrm{FW}}(s)+\mathrm{OC}_{\mathrm{W}}(s)\right) \\
& +\theta\left(\mathrm{VaR}+\frac{1}{1-\beta} \sum_{s=1}^{\mathrm{NS}} p(s)(f(s)-\mathrm{VaR})\right) \\
\text { s.t. } & \text { constraints }(1)-(41) .
\end{array}
$$




\subsubsection{Scenario Reduction}

It is acknowledged from numerical experiments that a well-controlled choice of sample size can significantly reduce computational time and improve the accuracy of solutions. Thus, where appropriate, a scenario reduction procedure can be applied to reduce the high computational load suffered as a result of handling the potentially prohibitively large number of scenarios generated. This procedure is particularly useful to facilitate the simulation to determine VaR. A number of scenario reduction methods are available in the literature, e.g., see the work of Romisch (2009) and a statistical-based approach employed in You et al. (2009). Further references are available on this subject (e.g., Law and Kelton (2000)).

\subsection{Global Optimization}

The model is nonconvex in the bilinear terms arising from contaminant mixing that may result in multiple local optima (Quesada and Grossmann, 1995). Thus, implementing a global optimization approach is required to obtain a certificate of optimality and reliability to the solution.

\subsubsection{Natural Variable Bounds}

For the natural bounds, nonnegativity conditions are enforced for all continuous variables: 


$$
\begin{aligned}
& F_{\mathrm{A}, \mathrm{B}}(\alpha, \beta, s) \geq 0, \\
& \forall(\alpha, \mathrm{A}) \in(I, \mathrm{SO}) \cup\left(K_{\mathrm{NM}}, \mathrm{NM}\right) \cup\left(K_{\mathrm{M}}, \mathrm{MP}\right) \cup\left(K_{\mathrm{M}}, \mathrm{MR}\right) \text {, } \\
& \forall(\beta, \mathrm{B}) \in\left(K_{\mathrm{NM}}, \mathrm{NM}\right) \cup\left(K_{\mathrm{M}}, \mathrm{MP}\right) \cup\left(K_{\mathrm{M}}, \mathrm{MR}\right) \cup(J, \mathrm{SI}) \text {, } \\
& \forall s \in S \text {, } \\
& F_{\mathrm{A}}^{\mathrm{F}}(k, s) \geq 0, \quad \forall(k, \mathrm{~A}) \in\left(K_{\mathrm{NM}}, \mathrm{NM}\right) \cup\left(K_{\mathrm{M}}, \mathrm{M}\right), \forall s \in S, \\
& C_{\mathrm{A}}(k, q, s) \geq 0, \quad \forall(k, \mathrm{~A}) \in\left(K_{\mathrm{M}}, \mathrm{MP}\right) \cup\left(K_{\mathrm{M}}, \mathrm{MR}\right), \forall q \in Q, \forall s \in S, \\
& C_{\mathrm{A}}^{\mathrm{F}}(k, q, s) \geq 0, \quad \forall(k, \mathrm{~A}) \in\left(K_{\mathrm{NM}}, \mathrm{NM}\right) \cup\left(K_{\mathrm{M}}, \mathrm{M}\right), \forall q \in Q, \forall s \in S
\end{aligned}
$$

The nonnegativity constraints also obviate the possibility of reverse flows in a single-choice interconnection.

For the $0-1$ integer variables, integrality conditions are enforced:

$$
y_{\mathrm{A}, \mathrm{B}}(\alpha, \beta) \in\{0,1\} \text {. }
$$

\subsubsection{Tighter Variable Bounds}

It is imperative to specify tight lower and upper bounds for all variables in solving nonconvex problems to obtain resulting tight relaxations. Both solvers of our choice (BARON and GloMIQO) share a common emphasis on the importance of supplying good variable bounds, 
mainly for constructing convex relaxations for the nonconvex bilinear terms in executing the solution procedure. We use equation-based variable upper bounds as summarized in Table 1 for a more rigorous representation in the same manner as Ahmetović and Grossmann (2011) and Misener and Floudas (2010). The variable lower bounds are taken to be the minimum physicallyfeasible flowrates for controllability or economical purposes, which can be taken as 2 ton/h (Chakraborty, 2009).

Table 2. Variable upper bounds

\begin{tabular}{|c|c|c|c|c|}
\hline Equation & Flow variable & Origin & Destination & Upper bound \\
\hline 26 & $F_{\mathrm{SO}, \mathrm{SI}}(i, j, s)$ & $\begin{array}{l}\text { Source except } \\
\text { freshwater }\end{array}$ & Sink & Minimum of the two entities \\
\hline 27 & $\begin{array}{l}F_{\mathrm{SO}, \mathrm{A}}(i, k, s) \text { where } \mathrm{A}= \\
\text { regenerator } \in\{\mathrm{NM}, \mathrm{MP}, \mathrm{MR}\}\end{array}$ & $\begin{array}{l}\text { Source except } \\
\text { freshwater }\end{array}$ & Regenerator & $\begin{array}{l}\text { Equal to the source flow } \\
F_{\mathrm{SO}}(i, s)\end{array}$ \\
\hline 28 & $F_{\mathrm{A}, \mathrm{SI}}(k, j, s)$ & Regenerator & Sink & Equal to the sink flow $F_{\mathrm{SI}}(j, s)$ \\
\hline 29 & $F_{\mathrm{A}, \mathrm{B}}\left(k, k^{\prime}, s\right)$ & Regenerator & $\begin{array}{l}\text { Other } \\
\text { regenerator }\end{array}$ & $\begin{array}{l}\text { Maximum of the total source } \\
\text { flows except freshwater and } \\
\text { total sink flows }\end{array}$ \\
\hline 30 & $\sum_{i \in I} \sum_{j \in J} F_{\mathrm{SO}, \mathrm{SI}}(i, j, s)+F_{\mathrm{A}, \mathrm{SI}}(k, j, s)$ & $\begin{array}{l}\text { Source and } \\
\text { regenerator }\end{array}$ & Discharge & $\begin{array}{l}\text { Equal to total source flows } \\
\Sigma F_{\mathrm{SO}}(i, s)\end{array}$ \\
\hline 31 & $F_{\mathrm{FW}}(s)$ & Freshwater & $\begin{array}{l}\text { Regenerators } \\
\text { and sinks }\end{array}$ & $\begin{array}{l}\text { Equal to total sink flows except } \\
\text { discharge }\end{array}$ \\
\hline 32 & $F_{\mathrm{W}}(s)$ & $\begin{array}{l}\text { Sources and } \\
\text { regenerators }\end{array}$ & Discharge & $\begin{array}{l}\text { Equal to total source flows } \\
\text { except freshwater }\end{array}$ \\
\hline
\end{tabular}

Note that the right-hand-side minimization operation involves computing the minimum value over the stipulated controlling indices of $i, j$, and $s$.

$F_{\mathrm{SO}, \mathrm{A}}^{\mathrm{U}}(i, k, s)=F_{\mathrm{SO}}(i, s), \quad \forall(k, \mathrm{~A}) \in\left(K_{\mathrm{NM}}, \mathrm{NM}\right) \cup\left(K_{\mathrm{M}}, \mathrm{MP}\right) \cup\left(K_{\mathrm{M}}, \mathrm{MR}\right), \forall i \in I, \forall s \in S$, 


$$
\begin{gathered}
F_{\mathrm{A}, \mathrm{SI}}^{\mathrm{U}}(k, j, s)=F_{\mathrm{SI}}(j, s), \quad \\
\forall(k, \mathrm{~A}) \in\left(K_{\mathrm{NM}}, \mathrm{NM}\right) \cup\left(K_{\mathrm{M}}, \mathrm{MP}\right) \cup\left(K_{\mathrm{M}}, \mathrm{MR}\right), \forall j \in J, \forall s \in S, \\
F_{\mathrm{A}, \mathrm{B}}^{\mathrm{U}}\left(k, k^{\prime}, s\right)=\max \left(\sum_{i \in I} F_{\mathrm{SO}}(i, s), \sum_{j \in J} F_{\mathrm{SI}}(j, s)\right), \\
\forall(k, \mathrm{~A}) \in\left(K_{\mathrm{NM}}, \mathrm{NM}\right) \cup\left(K_{\mathrm{M}}, \mathrm{MP}\right) \cup\left(K_{\mathrm{M}}, \mathrm{MR}\right), \\
\forall\left(k^{\prime}, \mathrm{B}\right) \in\left(K_{\mathrm{NM}}, \mathrm{NM}\right) \cup\left(K_{\mathrm{M}}, \mathrm{MP}\right) \cup\left(K_{\mathrm{M}}, \mathrm{MR}\right), \\
k \neq k^{\prime}, \forall s \in S, \\
\sum_{i \in I} \sum_{k \in K}\left(F_{\mathrm{SO}, \mathrm{SI}}^{\mathrm{U}}(i, j, s)+F_{\mathrm{A}, \mathrm{SI}}^{\mathrm{U}}(k, j, s)\right)=\sum_{i \in I} F_{\mathrm{SO}}(i, s), \quad j \in\{\text { Discharge }\}, \forall s \in S, \\
F_{\mathrm{FW}}^{\mathrm{U}}(s)=\sum_{j \in J \backslash\{\text { Discharge }\}} F_{\mathrm{SI}}(j, s), \quad \forall s \in S, \\
F_{\mathrm{W}}^{\mathrm{U}}(s)=\sum_{i \in I} F_{\mathrm{SO}}(i, s), \quad \forall s \in S .
\end{gathered}
$$

The concentration variables are generally bounded from above by the maximum source concentration for the corresponding contaminant $q$ :

$$
\begin{gathered}
C_{\mathrm{A}}^{\mathrm{F}, \mathrm{U}}(k, q, s)=\max _{\forall i \in I} C_{\mathrm{SO}}(i, q), \quad \forall(k, \mathrm{~A}) \in\left(K_{\mathrm{NM}}, \mathrm{NM}\right) \cup\left(K_{\mathrm{M}}, \mathrm{M}\right), \forall q \in Q, \forall s \in S, \\
C_{\mathrm{NM}}^{\mathrm{U}}(k, q, s)=\max _{\forall i \in I} C_{\mathrm{SO}}(i, q), \quad \forall k \in K, \forall q \in Q, \forall s \in S,
\end{gathered}
$$




$$
\begin{gathered}
C_{\mathrm{MP}}^{\mathrm{U}}(k, q, s)=(1-R(k, q, s)) \max _{\forall i \in I} C_{\mathrm{SO}}(i, q), \quad \forall k \in K, \forall q \in Q, \forall s \in S, \\
C_{\mathrm{MR}}^{\mathrm{U}}(k, q, s)=R(k, q, s) \max _{\forall i \in I} C_{\mathrm{SO}}(i, q), \quad \forall k \in K, \forall q \in Q, \forall s \in S .
\end{gathered}
$$

\subsubsection{Heuristic-Based Cuts}

We append the heuristic-based logic cuts introduced in Khor et al. (2012b) as additional constraints into the model to increase the convergence. These linear logical constraints are derived generically based on design and structural specifications of water network synthesis problems and may be applicable to related process synthesis problems.

Importantly, the model given by the objective function in equation (30) subject to constraints (9)-(31) corresponds to a risk-neutral formulation. In the next section, we present an extension of the formulation to handle risk management.

\section{Computational Results}

A numerical example is presented that is inspired from a real-life case study of a petroleum refinery water network in Malaysia. Seven water-using operations are involved that comprise three sources as well as freshwater and three sinks. Two types of water regeneration technologies are considered, namely a cartridge (carbon) filter and a single-stage reverse osmosis network (RON) regenerator with uncertain removal ratios for two contaminants, i.e., oil and grease 
$(\mathrm{O \& G})$ and total suspended solids (TSS). Data for the problem is given in Tables 2 to 5, which are invariant over all scenarios except for the freshwater and discharge flows, as indicated.

Table 3. Data on water sources

\begin{tabular}{lccc}
\hline & & \multicolumn{2}{c}{ Concentration $C_{\mathrm{SO}}(\mathrm{mg} / \mathrm{L})$} \\
\cline { 3 - 4 } \multicolumn{1}{c}{ Water Source $i$} & Flowrate $F_{\text {SO }}($ ton $/ \mathrm{h})$ & Oil and Grease & Total Suspended Solids \\
\hline Process area & 23.00 & 2.00 & 40.00 \\
Blowdown losses & 3.500 & 1.00 & 37.00 \\
Sidestream filter backwash losses & 1.800 & 1.00 & 37.00 \\
Freshwater & (variable) & 3.00 & 10.00 \\
\hline
\end{tabular}

Table 4. Data on water sinks

\begin{tabular}{lccc}
\hline & & \multicolumn{2}{c}{ Maximum Allowable Inlet Concentration (MAIC) (mg/L) } \\
\cline { 3 - 4 } \multicolumn{1}{c}{ Water Sink $j$} & Flowrate (ton/h) & Oil and Grease & Total Suspended Solids \\
\hline Cooling tower & 25.60 & 25.00 & 25.00 \\
Boiler & 115.0 & 0.10 & 10.00 \\
Desalter & 28.40 & 25.00 & 25.00 \\
Discharge & (variable) & 10.00 & 25.00 \\
\hline
\end{tabular}

Table 5. Data on cartridge filter regenerator

\begin{tabular}{lll}
\hline \multirow{2}{*}{ Contaminant } & \multicolumn{2}{c}{ Removal ratio } \\
\cline { 2 - 3 } & Lower limit & Upper limit \\
\hline Oil and grease & 0 & 0 \\
Total suspended solids & 0.63 & 0.77 \\
\hline
\end{tabular}

Table 6. Data on reverse osmosis network regenerator

\begin{tabular}{lrr}
\hline Split ratio based on recovery factor $\lambda$ & \multicolumn{2}{c}{0.7} \\
\cline { 2 - 3 } \multicolumn{1}{c}{ Contaminant } & \multicolumn{2}{c}{ Removal ratio } \\
\hline Oil and grease & 0.45 & Upper limit \\
Total suspended solids & 0.97 & 0.55 \\
\hline
\end{tabular}

Table 7. Economic data

\begin{tabular}{lr}
\hline \multicolumn{1}{c}{ Parameter } & Value \\
\hline Annual operating time $H$ & $8,760 \mathrm{~h} / \mathrm{y}$ \\
Unit cost for freshwater $C_{\mathrm{FW}}$ & $\$ 1.00 / \mathrm{t}$ \\
Unit cost for effluent treatment $C_{\mathrm{W}}$ & $\$ 1.00 / \mathrm{t}$ \\
Annualized capital charge factor $\delta$ & $0.1 / \mathrm{y}$ \\
Capital cost coefficient for purchasing individual piping $b_{\mathrm{A}, \mathrm{B}}(\alpha, \beta)$ & $\$ 100$
\end{tabular}




\subsection{Scenario Generation}

In actual practical situations, only a subset of the uncertain parameters is random. In this work, as alluded to earlier, we consider uncertainty in the left-hand side constraint coefficients of each of the removal ratios $R(k, q, s)$ for a contaminant $q$ of a regenerator $k$ in a scenario $s$. We apply a Monte Carlo-based pseudorandom number generation technique for the uncertain $R$ values that involves sampling of finitely many, mutually exclusive scenarios from a discrete distribution developed based on historical data analysis. Each scenario is assigned an equiprobability value for occurrence of $p_{s}$ equals to $1 / \mathrm{NS}$.

\subsection{Risk-Neutral Model Solution}

We solve the problems using a state-of-the-art commercial global solver, BARON 11.9.1 (Tawarmalani and Sahinidis, 2005) and a new commercial global MIQCQP solver, GloMIQO 2.1 (Misener and Floudas, 2012), both from GAMS 24.0.2. It is noteworthy that the purpose here is not to compare the performance of the two solvers, but rather to verify the solution obtained from one solver against the other in an independent manner.

Table 6 summarizes the model size and solution statistics in solving the risk-neutral model. Both BARON and GloMIQO may incorporate redundant constraints derived from the reformulationlinearization technique (RLT) (Sherali and Alameddine, 1992) to enhance computational 
procedure. The resulting network are then shown in Figure 5 and Figure 6 for solutions obtained using BARON and GloMIQO, respectively, for a relative optimality tolerance $\varepsilon_{r}$ of $10^{-3}(0.1 \%)$.

Table 8: Model size and computational statistics for the risk-neutral model with 100 scenarios

\begin{tabular}{|c|c|}
\hline Computing platform & 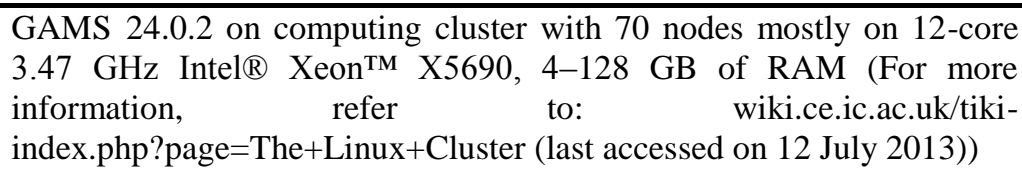 \\
\hline No. of continuous variables & 2498 \\
\hline No. of $0-1$ variables & 28 \\
\hline No. of constraints & 2207 (plus any RLT equation) \\
\hline No. of nonconvex bilinear terms & 900 \\
\hline
\end{tabular}

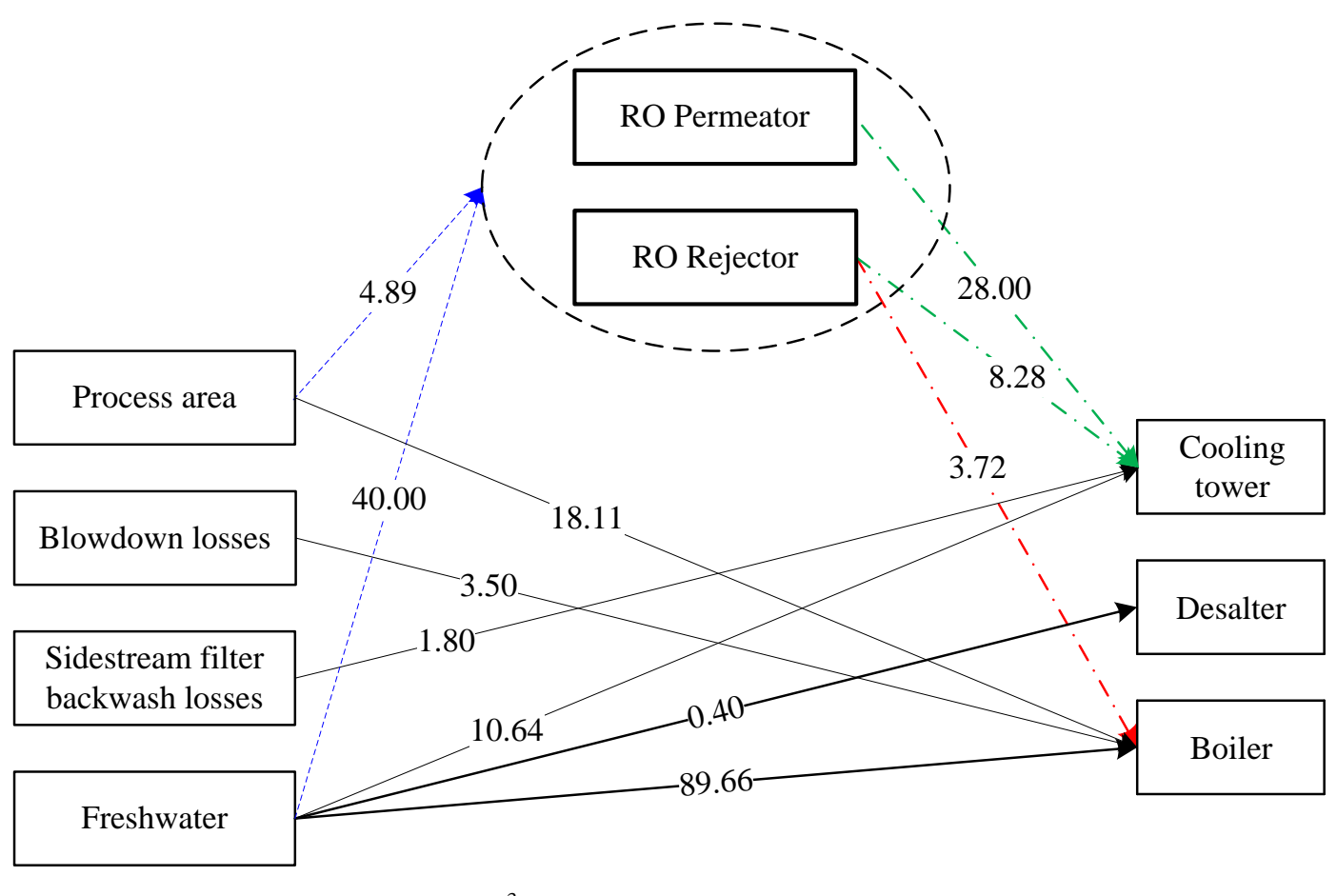

$\operatorname{BARON}\left(\varepsilon_{r}=10^{-3}\right):$ Total cost $=\$ 1,245,899 /$ year $(380.00 \mathrm{~s})$

Figure 5. $\varepsilon$-Optimal risk-neutral network computed by BARON for $\varepsilon_{r}=10^{-3}$ 
The results indicate that $\varepsilon_{r}=10^{-3}$ is not suitable because the optimal solutions reported are not consistent. Moreover, the optimum network obtained with BARON involve impractical reuse of a regenerated $\mathrm{RO}$ rejector flow to supply the high purity requirement (i.e., with low contaminant concentrations) of boiler feedwater (see Figure 5). Hence, there is a need to consider tighter tolerance to provide a certificate of global optimality (e.g., $\varepsilon_{r}=10^{-5}$ or $\varepsilon_{r}=10^{-6}$, instead of $\varepsilon_{r}=$ $10^{-3}$ ). In Figure 7, we present result for $\varepsilon_{r}=10^{-5}$ as obtained using GloMIQO since it generates a solution faster than BARON—interestingly, an optimal network suggests reuse of the permeator to supply the boiler feedwater. 


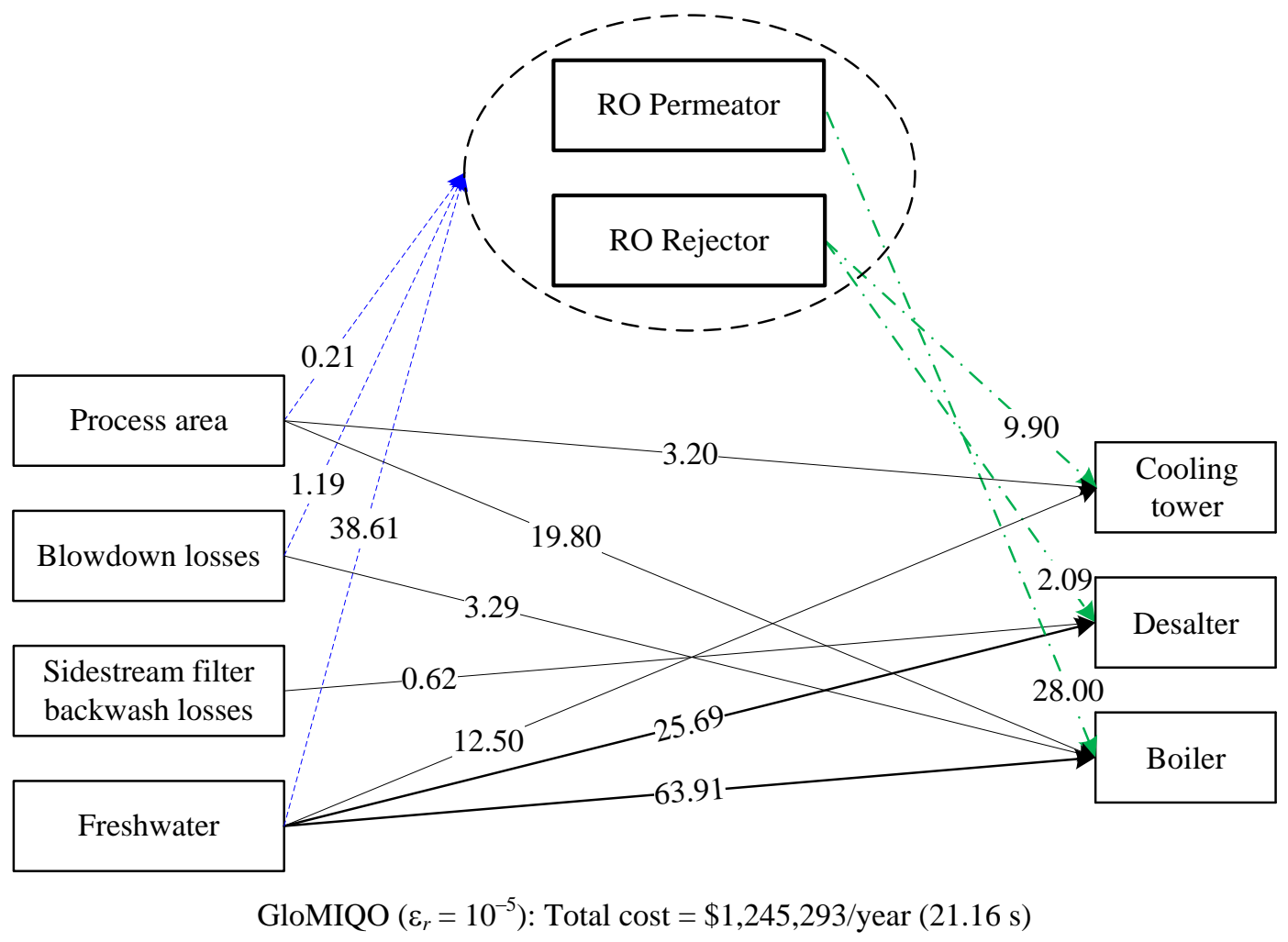

Figure 7. $\varepsilon$-Optimal risk-neutral network computed by GloMIQO for $\varepsilon_{r}=10^{-5}$

\subsection{Risk-Averse Model Solution}

For the purpose of prototyping, we employ manual intervention to estimate the VaR parameter from a CDF by plotting cumulative probability against the objective function value for each scenario (i.e., using the optimal risk-neutral solution). As shown in Figure 8, this involves a scenario-ordering procedure of sorting the values in ascending order to generate a CDF plot. The plot is utilized to determine $\mathrm{VaR}$ at a chosen confidence level of 95\% (i.e., at the corresponding $5 \%$ cumulative probability). It is noteworthy that this function evaluation procedure of CDF estimation for simulating VaR can be automated as necessary. While in principle, these are continuous curves as based on their integral-based definitions, they are presented here as discrete 
curves because the uncertain parameters are approximated through discretizing the parameter values to form the scenarios.

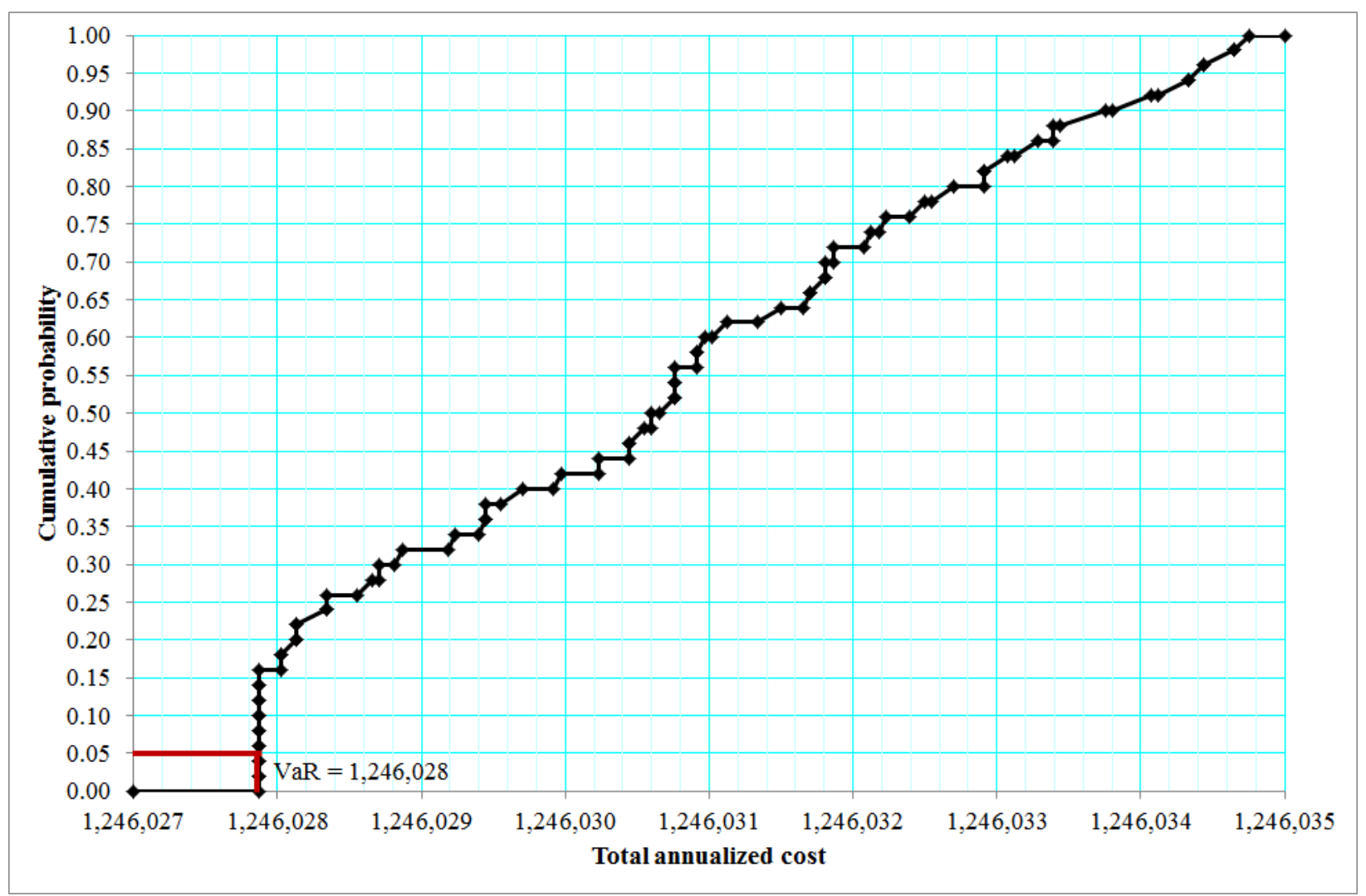

Figure 8. Cumulative distribution function (CDF) for determining VaR

In the final step, the risk-averse program with a mean-CVaR objective is solved to global optimality by accounting for a larger and more representative 100 scenarios. Table 9 summarizes the main parameters while Table 10 reports the model size and computational statistics.

Table 9. Parameters for risk-averse model

\begin{tabular}{lr}
\hline \multicolumn{1}{c}{ Parameter } & \multicolumn{1}{c}{ Value } \\
\hline No. of scenarios & 100 \\
Confidence level $\beta$ & 0.95 \\
$\mathrm{VaR}_{0.05}$ & $\$ 1,246,028 /$ year \\
\hline
\end{tabular}


Table 10: Model size and computational statistics for risk-averse model

\begin{tabular}{ll}
\hline Computing platform & GAMS 23.8.1 on Windows 7 on Sony Vaio laptop with Intel Core i7- \\
& 620M processor 2.66 GHz (with Turbo Boost up to 3.33 GHz) and 8 GB \\
& of RAM \\
No. of continuous variables & 4357 \\
No. of 0-1 variables & 28 \\
No. of constraints & 4948 and any RLT equation \\
No. of nonconvex bilinear terms & 1804 \\
\hline
\end{tabular}

Figure 9 and Figure 10 show the optimum network determined by GloMIQO for risk factors $\theta=1$ and $\theta=5$, respectively for $\varepsilon_{r}=10^{-5}$; similar results generally apply to other risk factors.

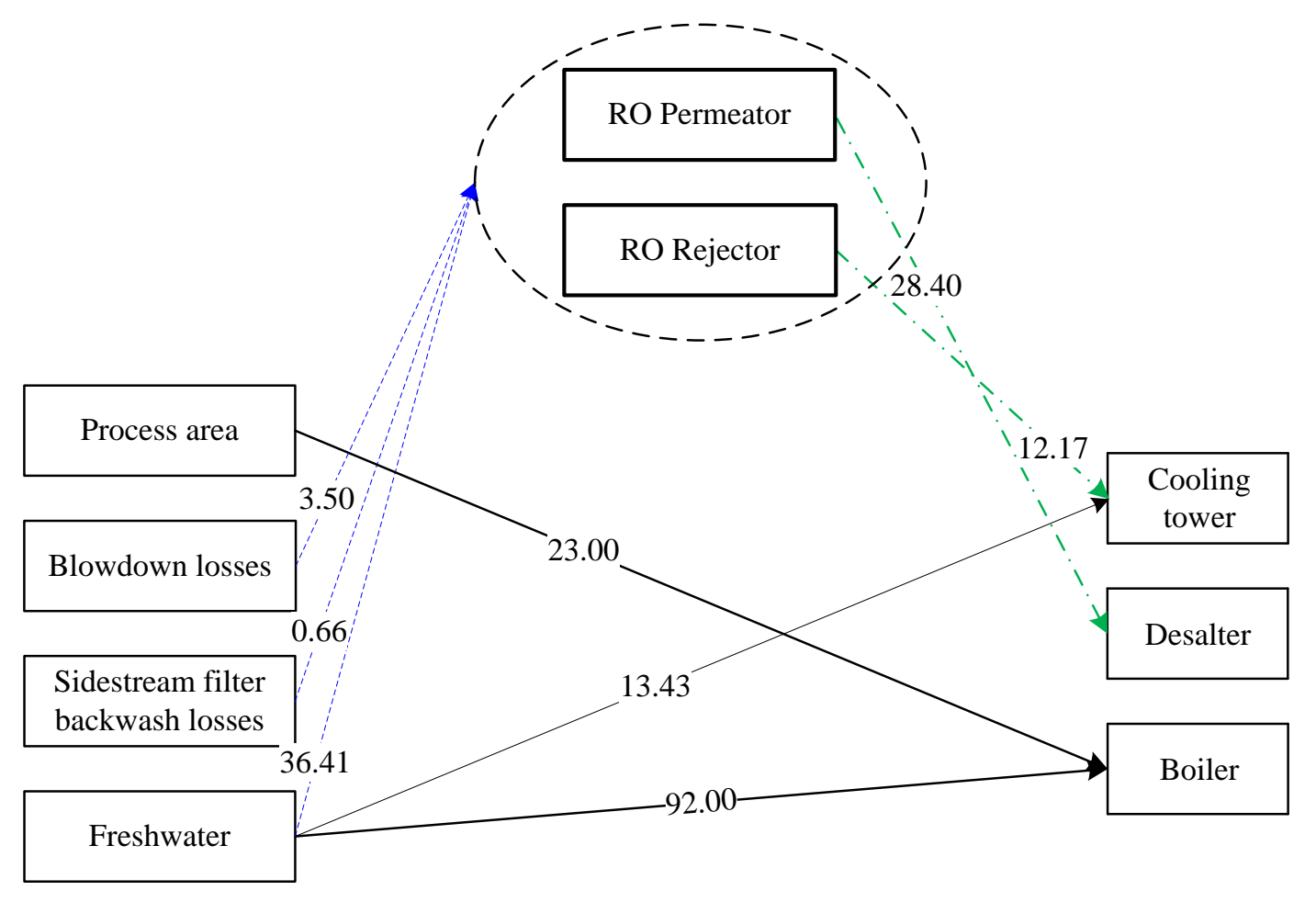

$$
\text { GloMIQO }\left(\varepsilon_{r}=10^{-5} ; \beta=0.95 ; \theta=1\right) \text { : Total cost }=\$ 1,250,042 / \text { year }(25.00 \mathrm{~s})
$$

Figure 9. $\varepsilon$-Optimal risk-averse network for risk factor $\theta=1\left(\varepsilon_{r}=10^{-5}\right)$ 


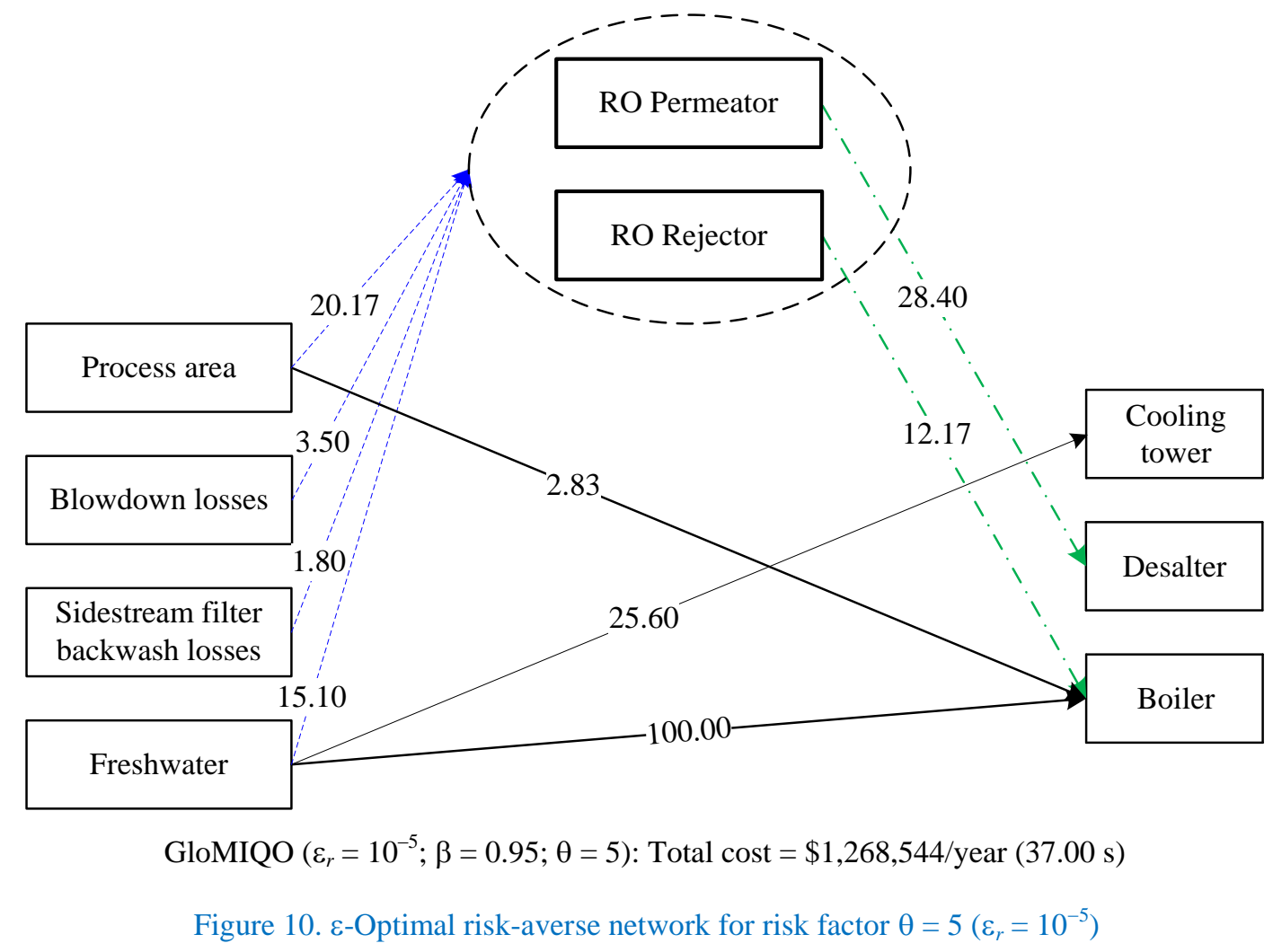

Finally, Figure 11 shows histograms illustrating the distributions of solutions for both the riskneutral and the risk-averse models as computed by GloMIQO for each of the 100 equiprobable scenarios for the instance of $\theta=1$. To investigate the incentive for considering risk, a comparison between the two model solutions reveal that while the expected total annualized cost (TAC) has increased for the risk-averse model, the risk of a higher or possibly very high TAC has been reduced compared to the risk-neutral model as shown in Figure 10 with similar trends observed for other risk factors. This result indicates that in accounting for risk, we gain in robustness (i.e., by reducing the risk of a very high total cost) but at the expense of a higher expected TAC, which is indeed intuitive (see also You et al. (2009)). Similar trends are observed for the results of other risk factors. 


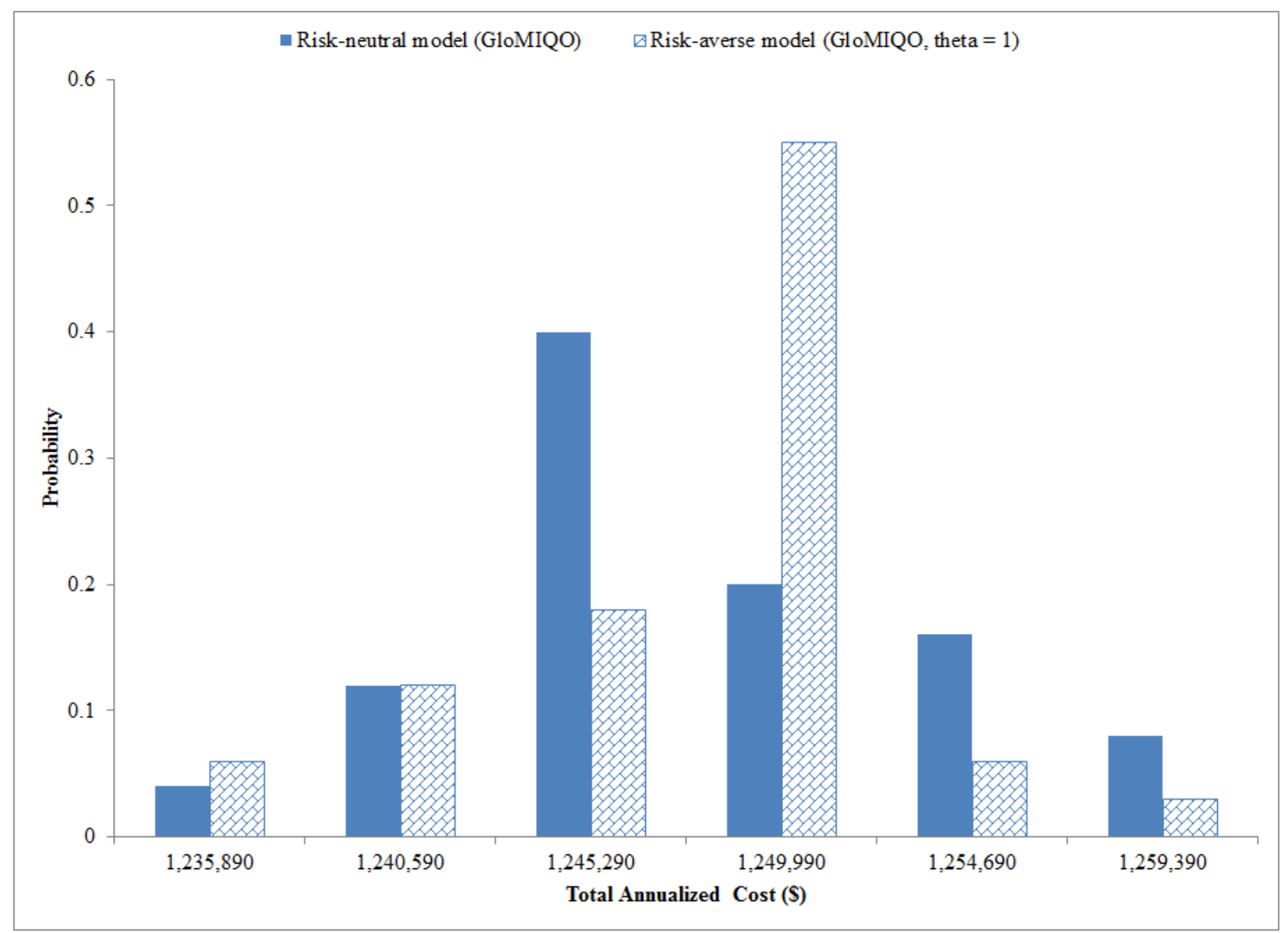

Figure 11. Histogram illustrating distribution of the risk-neutral and the risk-averse $(\theta=1)$ solutions computed by GloMIQO $\left(\varepsilon_{r}=10^{-5}\right)$

\section{Concluding Remarks}

The work in this article has presented an optimization-based framework for water network synthesis under uncertainty in contaminant removal ratios with risk management considerations. The proposed stepwise solution strategy involves solving risk-neutral and risk-averse formulations of recourse-based multiscenario two-stage stochastic MIQCQP. We first consider a risk-neutral model that offers a tractable approximate solution by using a small number of scenarios, which also consequently reduces the computational load in an ensuing scenarioordering scheme for simulating the value-at-risk (VaR) parameter. Ultimately, these steps feed 
into a risk-averse model that adopts a convex risk measure of conditional VaR (CVaR). We handle the model nonconvexities due to the presence of bilinear terms by employing GAMS 24.0.2/GloMIQO 1.0.0, a new global MIQCQP solver and verifying the solutions with GAMS 24.0.2/BARON 11.9.1, a state-of-the-art solver for the same problem class. Our computational experiments show that water network synthesis under uncertainty with risk management problems can be solved to global optimality in reasonable CPU time.

\section{Acknowledgment}

We sincerely thank Ruth Misener and Christodoulos A. Floudas for providing us with a free beta version of the GloMIQO license, as well as Michael R. Bussieck of GAMS Corporation for providing us with a free GAMS license that supports the GloMIQO library. Cheng Seong Khor is grateful for financial support from the Commonwealth Scholarship Plan, Centre for Process Systems Engineering (CPSE) of Imperial College London, Universiti Teknologi PETRONAS, and Ministry of Higher Education (MOHE) of Malaysia (Fundamental Research Grant Scheme (FRGS) number FRGS/1/10/TK/UTP/03/16).

\section{Notations}

Sets and Indices

I $\quad$ set of sources $i$

$J \quad$ set of sinks $j$

$K \quad$ set of all types of regenerators $k$ where $K=K_{\mathrm{M}} \cup K_{\mathrm{NM}}$

$K_{\mathrm{NM}} \quad$ set of non-membrane separation-based regenerators $k$

$K_{\mathrm{M}} \quad$ set of membrane separation-based regenerators $k$

$Q \quad$ set of contaminants $q$

Parameters 
concentration of contaminant $q$ in outlet of source $i$ in scenario $s(\mathrm{mg} / \mathrm{L})$ maximum concentration of contaminant $q$ at inlet to $\operatorname{sink} j(\mathrm{mg} / \mathrm{L})$ flow from outlet of source $i$ (ton $/ \mathrm{h}$ ) flow from outlet of freshwater source in scenario $s($ ton/h) flow to inlet of $\operatorname{sink} j$ (ton/h) flow to inlet of discharge sink in scenario $s$ (ton $/ \mathrm{h}$ ) annual operating time of the water systems plant (hour/year) removal ratio of contaminant $q$ in membrane regenerator $k$ (dimensionless) liquid-phase recovery factor of membrane regenerator $k$ (dimensionless) capital cost for purchasing of piping interconnection between an origin entity A (as indexed by $\alpha$ ) and a destination entity B (as indexed by $\beta$ ) (\$/ton)

$c_{\mathrm{A}, \mathrm{B}}(\alpha, \beta)$ operating cost of piping interconnection between an origin entity A (as indexed by $\alpha$ ) and a destination entity B (as indexed by $\beta$ ) (\$/ton)

$d_{\mathrm{A}, \mathrm{B}}(\alpha, \beta)$ capital cost coefficient for installation of piping interconnection between an origin entity A (as indexed by $\alpha$ ) and a destination entity B (as indexed by $\beta)(\$ /$ ton $)$

Continuous variables (flows in $\mathrm{t} / \mathrm{h}$, concentrations in $\mathrm{mg} / \mathrm{L}$ )

$F_{\mathrm{A}, \mathrm{B}}(\alpha, \beta, s) \quad$ flow from an origin entity A (as indexed by $\alpha$ ) and a destination entity B (as indexed by $\beta$ ) in scenario $s$

$F_{\mathrm{A}, \mathrm{B}}^{\max }(\alpha, \beta) \quad$ maximum flow from an origin entity $\mathrm{A}$ (as indexed by $\alpha$ ) and a destination entity B (as indexed by $\beta$ )

$F_{\mathrm{NM}}^{\mathrm{F}}(k, s)$

flow of feed stream to a non-membrane regenerator $k$ in scenario $s$

$F_{\mathrm{M}}^{\mathrm{F}}(k, s)$ flow of entry point to a membrane regenerator $k$ in scenario $s$

$C_{\mathrm{A}}^{\mathrm{F}}(k, q, s)$

$C_{\mathrm{NM}}(k, q, s)$ concentration of contaminant $q$ in feed stream to non-membrane regenerator $k$ (or entry point to membrane regenerator) in scenario $s$ concentration of contaminant $q$ in outlet of non-membrane regenerator $k$ in scenario $s$

$C_{\mathrm{MP}}(k, q, s)$ concentration of contaminant $q$ in outlet of permeator of membrane regenerator $k$ in scenario $s$

$C_{\mathrm{MR}}(k, q, s) \quad$ concentration of contaminant $q$ in outlet of rejector of membrane regenerator $k$ in scenario $s$

\section{Binary variables}

$y_{\mathrm{A}, \mathrm{B}}(\alpha, \beta) \quad$ existence of interconnection from an origin entity A (as indexed by $\alpha$ ) and a destination entity B (as indexed by $\beta$ ) 


\section{Subscripts}

$\begin{array}{ll}\text { NM } & \text { index for non-membrane regenerators } \\ \text { MP } & \text { index for permeator of membrane regenerators } \\ \text { MR } & \text { index for rejector of membrane regenerators }\end{array}$

\section{References}

Ahmetović, E., Grossmann, I.E., 2011. Global superstructure optimization for the design of integrated process water networks. AIChE J 57, 434-457.

Al-Redhwan, S.A., Crittender, B.D., Lababidi, H.M.S., 2005. Wastewater minimization under uncertain operational conditions. Comput Chem Eng 29, 1009-1021.

Bakker, K.J., 2010. Privatizing Water: Governance Failure and the World's Urban Water Crisis. Cornell University Press, Ithaca, NY.

Barbaro, A., Bagajewicz, M.J., 2004. Managing financial risk in planning under uncertainty. AIChE J 50, 963-989.

Chakraborty, A., 2009. A globally convergent mathematical model for synthesizing topologically constrained water recycle networks. Computers and Chemical Engineering 33, 1279-1288.

Chang, C.T., Li, B.H., Liou, C.W., 2009. Development of a Generalized Mixed Integer Nonlinear Programming Model for Assessing and Improving the Operational Flexibility of Water Network Designs. Ind Eng Chem Res 48, 3496-3504.

Colvin, M., Maravelias, C.T., 2011. R\&D pipeline management: Task interdependencies and risk management. Eur J Oper Res 215, 616-628.

Eppen, G.D., Martin, R.K., Schrage, L., 1989. A Scenario Approach to Capacity Planning. Oper Res 37, 517-527.

Foo, D.C.Y., 2009. State-of-the-Art Review of Pinch Analysis Techniques for Water Network Synthesis. Ind Eng Chem Res 48, 5125-5159.

Goel, V., Grossmann, I.E., 2006. A Class of stochastic programs with decision dependent uncertainty. Math Program 108, 355-394.

Hung, S.W., Kim, J.K., 2011. Integrated water networks optimisation under uncertainty. Chem Eng J 175, 56-69.

International Water Management Institute (IWMI), 2007. Water for Food, Water for Life: A Comprehensive Assessment of Water Management in Agriculture. Earthscan London, UK.

Jonsbraten, T.W., Wets, R.J.B., Woodruff, D.L., 1998. A class of stochastic programs with decision dependent random elements. Ann Oper Res 82, 83-106.

Karuppiah, R., Grossmann, I.E., 2008. Global optimization of multiscenario mixed integer nonlinear programming models arising in the synthesis of integrated water networks under uncertainty. Comput Chem Eng 32, 145-160.

Khor, C.S., Abang Zamhari, N., Nadzmi, S.N., 2009. A mathematical modeling approach to water reuse, regeneration, and recycle initiatives in PETRONAS Penapisan (Melaka) Sdn. Bhd (PP(M)SB), 4th PETRONAS Water Technology Conference 2009, Subang, Selangor, Malaysia.

Khor, C.S., Chachuat, B., Shah, N., 2012a. Optimal water network synthesis with detailed membrane-based regenerator models, in: Karimi, I.A., Srinivasan, R. (Eds.), Computer Aided Chemical Engineering, pp. 1457-1461.

Khor, C.S., Chachuat, B., Shah, N., 2012b. A superstructure optimization approach for water network synthesis with membrane separation-based regenerators. Comput Chem Eng 42, 48-63. 

Optimization Approach for Membrane Separation-Based Water Regeneration Network Synthesis with Detailed Nonlinear Mechanistic Reverse Osmosis Model. Ind Eng Chem Res 50, 1344413456.

Khor, C.S., Giarola, S., Chachuat, B., Shah, N., 2011. An Optimization-Based Framework for Process Planning under Uncertainty with Risk Management. Chemical Product and Process Modeling 6, Article 4.

Koppol, A.P.R., Bagajewicz, M.J., 2003. Financial risk management in the design of water utilization systems in process plants. Ind Eng Chem Res 42, 5249-5255.

Law, A.M., Kelton, W.D., 2000. Simulation Modeling and Analysis, 3rd ed. McGraw-Hill, New York.

Li, B.H., Chang, C.T., 2011. Efficient Flexibility Assessment Procedure for Water Network Designs. Ind Eng Chem Res 50, 3763-3774.

Li, X., Armagan, E., Tomasgard, A., Barton, P.I., 2011. Stochastic Pooling Problem for Natural Gas Production Network Design and Operation Under Uncertainty. AIChE J 57, 2120-2135.

Lim, S.R., Kim, Y.R., Woo, S.H., Park, D., Park, J.M., 2013. System optimization for eco-design by using monetization of environmental impacts: a strategy to convert bi-objective to singleobjective problems. J Clean Prod 39, 303-311.

Maskan, F., Wiley, D.E., Johnston, L.P.M., Clements, D.J., 2000. Optimal design of reverse osmosis module networks. AIChE J 46, 946-954.

McCormick, G.P., 1976. Computability of Global Solutions to Factorable Nonconvex Programs 1: Convex Underestimating Problems. Math Program 10, 147-175.

Misener, R., Floudas, C., 2012. GloMIQO: Global mixed-integer quadratic optimizer. J Global Optim, 48 pages.

Misener, R., Floudas, C.A., 2010. Global Optimization of Large-Scale Generalized Pooling Problems: Quadratically Constrained MINLP Models. Ind Eng Chem Res 49, 5424-5438.

Quesada, I., Grossmann, I.E., 1995. Global Optimization of Bilinear Process Networks with Multicomponent Flows. Comput Chem Eng 19, 1219-1242.

Riyanto, E., Chang, C.T., 2010. A heuristic revamp strategy to improve operational flexibility of water networks based on active constraints. Chem Eng Sci 65, 2758-2770.

Rockafellar, R.T., Uryasev, S., 2000. Optimization of Conditional Value-at-Risk. Journal of Risk $2,21-41$.

Rockafellar, R.T., Uryasev, S., 2002. Conditional value-at-risk for general loss distributions. J Bank Financ 26, 1443-1471.

Romisch, W., 2009. Scenario Reduction Techniques in Stochastic Programming. Lect Notes Comput Sc 5792, 1-14.

Santoso, T., Ahmed, S., Goetschalckx, M., Shapiro, A., 2005. A stochastic programming approach for supply chain network design under uncertainty. Eur J Oper Res 167, 96-115. Schultz, R., 2003. Stochastic programming with integer variables. Math Program 97, 285-309. Sen, S., Higle, J.L., 1999. An introductory tutorial on stochastic linear programming models. Interfaces 29, 33-61.

Sherali, H.D., Alameddine, A., 1992. A new reformulation linearization technique for bilinear programming problems. Journal of Global Optimization 2, 379-410.

Sotelo-Pichardo, C., Ponce-Ortega, J.M., El-Halwagi, M.M., Frausto-Hernandez, S., 2011. Optimal retrofit of water conservation networks. J Clean Prod 19, 1560-1581.

Szego, G., 2002. Measures of risk. Journal of Banking \& Finance 26, 1253-1272. 
Tan, R.R., Foo, D.C.Y., Manan, Z.A., 2007. Assessing the sensitivity of water networks to noisy mass loads using Monte Carlo simulation. Comput Chem Eng 31, 1355-1363.

Tan, R.R., Ng, D.K.S., Foo, D.C.Y., Aviso, K.B., 2009. A superstructure model for the synthesis of single-contaminant water networks with partitioning regenerators. Process Saf Environ 87, 197-205.

Tarhan, B., Grossmann, I.E., Goel, V., 2009. Stochastic Programming Approach for the Planning of Offshore Oil or Gas Field Infrastructure under Decision-Dependent Uncertainty. Ind Eng Chem Res 48, 3078-3097.

Tawarmalani, M., Sahinidis, N.V., 2005. A polyhedral branch-and-cut approach to global optimization. Math Program 103, 225-249.

Tchobanoglous, G., Burton, F.L., Stensel, H.D., 2004. Wastewater Engineering: Treatment and Reuse (Metcalf \& Eddy), 4th ed. McGraw-Hill, New York, NY.

Tokos, H., Pintaric, Z.N., Yang, Y.R., 2013. Bi-objective optimization of a water network via benchmarking. J Clean Prod 39, 168-179.

Tometzki, T., Engell, S., 2011. Risk conscious solution of planning problems under uncertainty by hybrid multi-objective evolutionary algorithms. Comput Chem Eng 35, 2521-2539.

UNEP, 2008. Vital Water Graphics: An Overview of the State of the World's Fresh and Marine Waters, 2nd ed. UNEP, Nairobi, Kenya.

Verderame, P.M., Floudas, C.A., 2010. Operational Planning of Large-Scale Industrial Batch Plants under Demand Due Date and Amount Uncertainty: II. Conditional Value-at-Risk Framework. Ind Eng Chem Res 49, 260-275.

Verderame, P.M., Floudas, C.A., 2011. Multisite Planning under Demand and Transportation Time Uncertainty: Robust Optimization and Conditional Value-at-Risk Frameworks. Ind Eng Chem Res 50, 4959-4982.

Webby, R.B., Adamson, P.T., Boland, J., Howlett, P.G., Metcalfe, A.V., Piantadosi, J., 2007. The Mekong-applications of value at risk (VAR) and conditional value at risk (CVAR) simulation to the benefits, costs and consequences of water resources development in a large river basin. Ecol Model 201, 89-96.

You, F.Q., Wassick, J.M., Grossmann, I.E., 2009. Risk Management for a Global Supply Chain Planning Under Uncertainty: Models and Algorithms. AIChE J 55, 931-946.

Yunt, M., Chachuat, B., Mitsos, A., Barton, P.I., 2008. Designing man-portable power generation systems for varying power demand. AIChE J 54, 1254-1269.

Zimmerman, J.B., Mihelcic, J.R., Smith, J., 2008. Global stressors on water quality and quantity. Environ Sci Technol 42, 4247-4254. 